\title{
Enantioselective Total Synthesis of Mollebenzylanol A
}

Yuichiro Kawamoto, Fuka Karube, Toyoharu Kobayashi, and Hisanaka Ito*

School of Life Sciences, Tokyo University of Pharmacy and Life Sciences, 1432-1

Horinouchi, Hachioji, Tokyo 192-0392, Japan

itohisa@toyaku.ac.jp

\section{Table of Contents}

$\begin{array}{ll}\text { Experimental procedure } & \mathrm{S} 2\end{array}$

${ }^{1} \mathrm{H}$ and ${ }^{13} \mathrm{C}$ NMR spectra of new compounds $\quad$ S13

$\begin{array}{ll}\text { X-Ray Crystallography Data for compounds: 15, } 20 & \text { S25 }\end{array}$ 


\section{General}

All reactions involving air- and moisture-sensitive reagents were carried out using standard syringe-septum cap techniques. Unless otherwise noted, all solvents and reagents were obtained from commercial suppliers and used without further purification. Routine monitoring of reactions was carried out Merck silica gel 60 F254 TLC plates. Column chromatography was performed on Kanto Chemical Silica Gel 60N (spherical, neutral 60-230 $\mu \mathrm{m}$ ) with the solvents indicated. Melting points were taken on a Yanako MP-S3 micro melting point apparatus and are uncorrected. ${ }^{1} \mathrm{H}$ and ${ }^{13} \mathrm{C}$ NMR spectra were measured with a JASCO ECZ $400 \mathrm{~S}$ (400 MHz) spectrometer. Chemical shifts were expressed in ppm using $\mathrm{CHCl}_{3}\left(7.26 \mathrm{ppm}\right.$ for ${ }^{1} \mathrm{H}$ NMR, 77.0 ppm for ${ }^{13} \mathrm{C}$ NMR) in $\mathrm{CDCl}_{3}, \mathrm{C}_{6} \mathrm{H}_{6}$ (7.16 ppm for ${ }^{1} \mathrm{H}$ NMR, $128.0 \mathrm{ppm}$ for ${ }^{13} \mathrm{C} \mathrm{NMR}$ ) in $\mathrm{C}_{6} \mathrm{D}_{6}$ and $\mathrm{CH}_{3} \mathrm{OH}$ (3.31 ppm for ${ }^{1} \mathrm{H}$ NMR, $49.1 \mathrm{ppm}$ for ${ }^{13} \mathrm{C} \mathrm{NMR}$ ) in $\mathrm{CD}_{3} \mathrm{OD}$ as internal standard. Infrared spectral measurements were carried out with a JASCO FT/IR-4700 and only noteworthy absorptions were listed. HRMS spectra measured on a Micromass LCT spectrometer. X-ray crystallographic analysis was taken with Burker APEX2 Ultra TXS. Oil bath was used in reactions that require heating and each temperature indicates oil bath temperature.

(S)-3-hydroxy-2,2-dimethylcyclopentan-1-one (11)

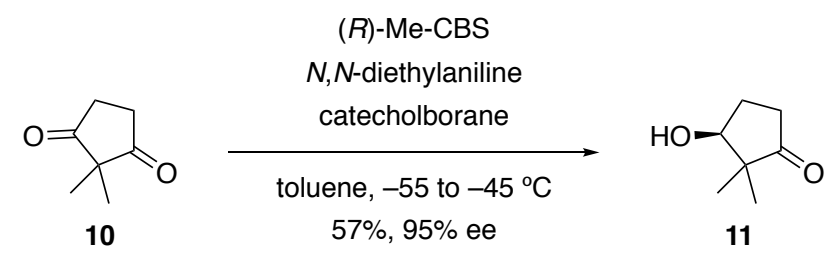

To a solution of 2,2-dimethylcyclopentane-1,3-dione $(\mathbf{1 0}, 700 \mathrm{mg}, 5.55 \mathrm{mmol})$ in toluene $(21$ $\mathrm{mL})$ was added $(R)$-Me-CBS $(1 \mathrm{M}$ in toluene, $0.56 \mathrm{ml}, 0.56 \mathrm{mmol})$ and $N, N$-diethylaniline $(0.44$ $\mathrm{mL}, 2.82 \mathrm{mmol}$ ) under Ar. The resulting solution was cooled to $-55{ }^{\circ} \mathrm{C}$ and a solution of catecholborane (1 $\mathrm{M}$ in toluene, $9.71 \mathrm{ml}, 9.71 \mathrm{mmol})$ was added slowly along the side of the reaction flask by using a syringe pump over $2 \mathrm{~h}$ with vigorous stirring. Following the addition, the temperature was allowed to raise to $-45{ }^{\circ} \mathrm{C}$ over $10 \mathrm{~min}$, stirring was continued for $30 \mathrm{~min}$, $\mathrm{MeOH}(1 \mathrm{~mL})$ was added slowly down the side wall of the flask and the solution was allowed to warm to room temperature. The resulting solution was diluted with $\mathrm{Et}_{2} \mathrm{O}(2 \mathrm{ml})$ and vigorously 
stirred with saturated $\mathrm{NaHCO}_{3}(7 \mathrm{~mL})$ and $3 \mathrm{M} \mathrm{NaOH}(7 \mathrm{~mL})$ for $2 \mathrm{~h}$. The mixture was extracted with $\mathrm{CH}_{2} \mathrm{Cl}_{2}$ (three times). The combined organic layers were washed with brine, dried over $\mathrm{MgSO}_{4}$, and concentrated in vacuo. The resulting residue was purified by silica gel column chromatography (hexane-AcOEt, 4:1) to afford 11 (406 mg, 57\%, 95\% ee) as colorless oil. Enantioselectivity was determined by the ${ }^{1} \mathrm{H}$ NMR analysis of the corresponding $(S)$-MTPA ester.

$[\alpha]_{\mathrm{D}}^{25}+8.13\left(c 0.27, \mathrm{CHCl}_{3}\right)$.

(S)-3-((tert-butyldimethylsilyl)oxy)-2,2-dimethylcyclopentan-1-one (S1)

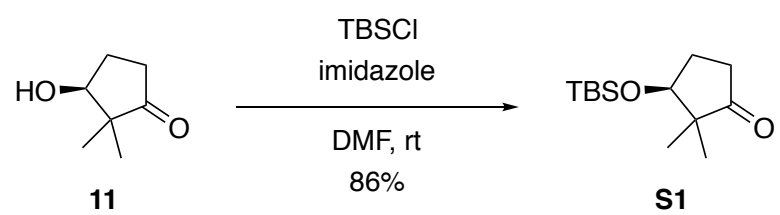

To a solution of $\mathbf{1 1}(1.23 \mathrm{~g}, 9.60 \mathrm{mmol})$ in DMF (48 mL) was added imidazole $(1.96 \mathrm{~g}, 28.8$ mmol) and TBSCl $(2.89 \mathrm{~g}, 19.2 \mathrm{mmol})$, and the mixture was stirred for $19 \mathrm{~h}$ at room temperature. The reaction mixture was quenched with $\mathrm{H}_{2} \mathrm{O}$, and extracted with $\mathrm{Et}_{2} \mathrm{O}$ (three times). The combined organic layers were washed with brine, dried over $\mathrm{MgSO}_{4}$, and concentrated in vacuo. The resulting residue was purified by silica gel column chromatography (hexane-AcOEt, 20:1) to afford S1 (2.01 g, 86\%) as colorless oil.

$[\alpha]_{\mathrm{D}}{ }^{25}+22.41\left(c 0.27, \mathrm{CHCl}_{3}\right)$.

(S)-4-((tert-butyldimethylsilyl)oxy)-5,5-dimethylcyclopent-2-en-1-one (8)

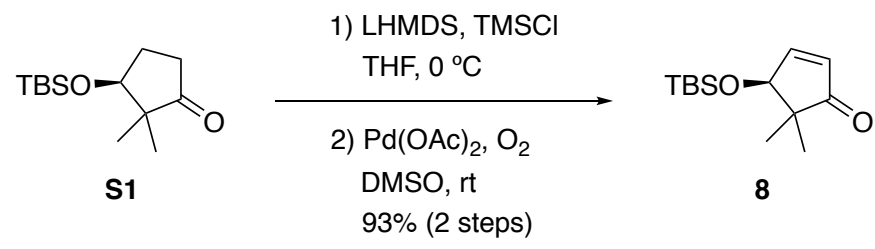

To a stirred solution of LHMDS (1 M in THF, $12.4 \mathrm{~mL}, 12.4 \mathrm{mmol})$ in THF (33 mL) was added dropwise $\mathbf{S 1}(2 \mathrm{~g}, 8.25 \mathrm{mmol})$ in THF $(8 \mathrm{~mL})$ at $0{ }^{\circ} \mathrm{C}$ under $\mathrm{Ar}$, and the reaction mixture was stirred for $1 \mathrm{~h}$ at $-78{ }^{\circ} \mathrm{C}$. To the mixture was added dropwise $\mathrm{TMSCl}(1.6 \mathrm{~mL}, 12.6 \mathrm{mmol})$ at 0 ${ }^{\circ} \mathrm{C}$, and the mixture was stirred for $1 \mathrm{~h}$ at the same temperature. The reaction mixture was quenched with cooled sat. $\mathrm{NH}_{4} \mathrm{Cl}$ aqueous solution, and extracted with AcOEt (twice). The combined organic layers were washed with brine, dried over $\mathrm{MgSO}_{4}$, and concentrated in vacuo. To the solution of crude product in DMSO $(15 \mathrm{~mL})$ was added $\mathrm{Pd}(\mathrm{OAc})_{2}(555 \mathrm{mg}, 2.47 \mathrm{mmol})$, and the mixture was stirred for $18 \mathrm{~h}$ at room temperature under $\mathrm{O}_{2}$ atmosphere. The reaction 
mixture was quenched with $\mathrm{H}_{2} \mathrm{O}$ and extracted with AcOEt (three times). The combined organic layers were washed with brine, dried over $\mathrm{MgSO}_{4}$, and concentrated in vacuo. The resulting residue was purified by silica gel column chromatography (hexane-AcOEt, 40:1) to afford 8 $(1.85 \mathrm{~g}, 93 \%)$ as yellow oil. $[\alpha]_{\mathrm{D}}^{25}+91.6\left(c 0.26, \mathrm{CHCl}_{3}\right)$.

(1S,4S)-4-((tert-butyldimethylsilyl)oxy)-5,5-dimethyl-1-(m-tolylethynyl)cyclopent-2-en-1-ol

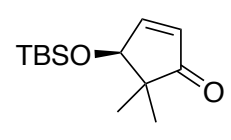

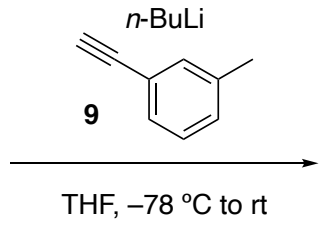

$89 \%, \mathrm{dr}>95: 5$

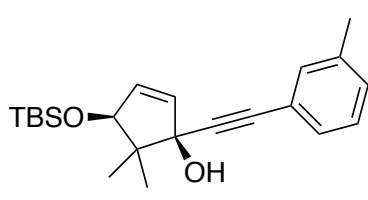

12

To a stirred solution of $n$-BuLi (1.59 M in $n$-hexane, $7.43 \mathrm{~mL}, 11.8 \mathrm{mmol})$ in THF (14 mL) was added dropwise 3-ethynyltoluene $(9,1.6 \mathrm{ml}, 12.5 \mathrm{mmol})$ at $-78{ }^{\circ} \mathrm{C}$ under $\mathrm{Ar}$, and the reaction mixture was stirred for $1.5 \mathrm{~h}$ at $-78^{\circ} \mathrm{C}$. To the mixture was added dropwise a solution of 8 (1.67 $\mathrm{g}, 6.95 \mathrm{mmol})$ in THF $(7 \mathrm{~mL})$ at $-78{ }^{\circ} \mathrm{C}$, and the mixture was stirred for $13 \mathrm{~h}$ at room temperature. The reaction mixture was quenched with sat. $\mathrm{NH}_{4} \mathrm{Cl}$ aqueous solution, and extracted with $\mathrm{Et}_{2} \mathrm{O}$ (twice). The combined organic layers were washed with brine, dried over $\mathrm{MgSO}_{4}$, and concentrated in vacuo. The resulting residue was purified by silica gel column chromatography (hexane-AcOEt, 40:1) to afford $12(2.21 \mathrm{~g}, 89 \%)$ as yellow solid. The solid was formed by concentration with hexane in vacuo.

Mp 52-54 ${ }^{\circ} \mathrm{C} .[\alpha]_{\mathrm{D}}{ }^{25}+235.61\left(c 0.29, \mathrm{CHCl}_{3}\right)$. IR (KBr): 3853, 3801, 3734, 3098, 2736, 2708, 2224, 1936, 1783, 1687, 732, 714, $625 \mathrm{~cm}^{-1} .{ }^{1} \mathrm{H} \mathrm{NMR}\left(\mathrm{CDCl}_{3}, 400 \mathrm{MHz}\right): \delta 7.27$ - 7.24 (m, 2H), 7.18 (t, 1H, $J=7.5 \mathrm{~Hz}), 7.12(\mathrm{~d}, 1 \mathrm{H}, J=7.8 \mathrm{~Hz}), 6.13$ (d, $1 \mathrm{H}, J=5.9 \mathrm{~Hz}), 5.96$ (dd, $1 \mathrm{H}, J=5.5$, $2.3 \mathrm{~Hz}$ ), 4.21 (d, 1H, J = 2.3 Hz), 2.32 (s, 3H), 1.17 (s, 3H), 1.13 (s, 3H), 0.89 (s, 9H), 0.10 (s, $3 \mathrm{H}), 0.08(\mathrm{~s}, 3 \mathrm{H})$, peak of $-\mathrm{OH}$ does not exist because of exchange to $\mathrm{CDCl}_{3}$ deuterium. ${ }^{13} \mathrm{C}$ NMR $\left(\mathrm{CDCl}_{3}, 400 \mathrm{MHz}\right): \delta 138.6,138.0,135.8,132.5,129.3,129.0,128.2,122.7,87.7,86.3$, 82.7, 81.7, 51.0, 25.9, 25.5, 21.3, 18.2, 17.3, -4.3, -4.8. (ESI-TOF) $\mathrm{m} / \mathrm{z}:[\mathrm{M}+\mathrm{Na}]^{+}$calcd for $\mathrm{C}_{22} \mathrm{H}_{32} \mathrm{O}_{2} \mathrm{SiNa}$ 379.2069; Found 379.2061.

$(1 R, 4 S)$-4-((tert-butyldimethylsilyl)oxy)-5,5-dimethyl-1-((Z)-3-methylstyryl)cyclopent-2-en-1-o 1 (13) 


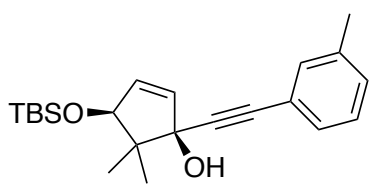

12

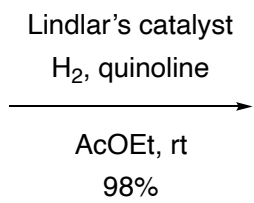

$98 \%$

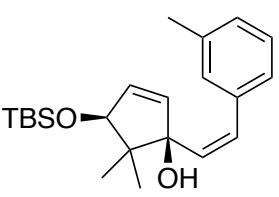

13

To a solution of $12(2.06 \mathrm{~g}, 5.78 \mathrm{mmol})$ and quinoline $(0.09 \mathrm{ml}, 0.762 \mathrm{mmol})$ in AcOEt $(29 \mathrm{~mL})$ was added Lindlar's catalyst (5\% on Calcium Carbonate poisoned with lead $638 \mathrm{mg}, 0.300$ mmol), and the mixture was stirred for $6 \mathrm{~h}$ at room temperature under $\mathrm{H}_{2}$ atmosphere. The insoluble material was filtered and concentrated in vacuo. The resulting residue was purified by silica gel column chromatography (hexane-AcOEt, 60:1) to afford 13 (2.03 g, 98\%) as colorless oil.

$[\alpha]_{\mathrm{D}}^{25}+104.99\left(c 0.29, \mathrm{CHCl}_{3}\right)$. IR (neat): 3583, 3515, 2955, 2929, 2895, 2857, 1470, 1254, 1089, 1049, 880, 836, $776 \mathrm{~cm}^{-1} .{ }^{1} \mathrm{H}$ NMR ( $\left.\mathrm{CDCl}_{3}, 400 \mathrm{MHz}\right): \delta$ 7.22-7.16 (m, 3H), $7.05(\mathrm{~d}, 1 \mathrm{H}$, $J=7.3 \mathrm{~Hz}), 6.61(\mathrm{~d}, 1 \mathrm{H}, J=12.8 \mathrm{~Hz}), 5.87(\mathrm{~d}, 1 \mathrm{H}, J=12.8 \mathrm{~Hz}), 5.71(\mathrm{dd}, 1 \mathrm{H}, J=5.5,2.3 \mathrm{~Hz})$, 5.59 (d, 1H, $J=12.8 \mathrm{~Hz}), 4.11(\mathrm{~d}, 1 \mathrm{H}, J=1.8 \mathrm{~Hz}), 2.33$ (s, 3H), $1.06(\mathrm{~s}, 3 \mathrm{H}), 0.99$ (s, 3H), 0.88 $(\mathrm{s}, 9 \mathrm{H}), 0.07(\mathrm{~s}, 3 \mathrm{H}), 0.04(\mathrm{~s}, 3 \mathrm{H})$, peak of $-\mathrm{OH}$ does not exist because of exchange to $\mathrm{CDCl}_{3}$ proton. ${ }^{13} \mathrm{C} \mathrm{NMR}\left(\mathrm{CDCl}_{3}, 400 \mathrm{MHz}\right): \delta 138.9,137.6,137.4,133.7,131.2,130.2,129.9,127.9$, $127.8,126.3,86.2,83.1,50.4,25.9,24.4,21.6,18.2,17.6,-4.3,-4.8$. HRMS (ESI-TOF) m/z: $[\mathrm{M}+\mathrm{Na}]^{+}$calcd for $\mathrm{C}_{22} \mathrm{H}_{34} \mathrm{O}_{2} \mathrm{SiNa} 381.2226$; Found 381.2219.

$(1 R, 4 S)-4-(($ tert-butyldimethylsilyl)oxy)-5,5-dimethyl-1-((2R,3R)-3-(m-tolyl)oxiran-2-yl)cyclop ent-2-en-1-ol (14)

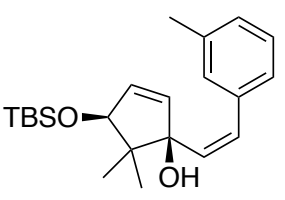

13

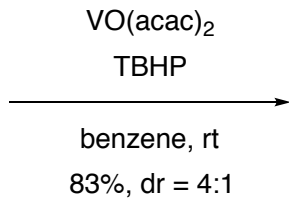

$83 \%, d r=4: 1$

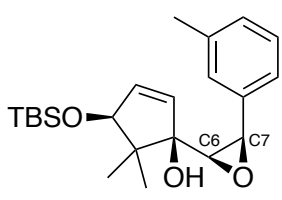

14

(diastereomer mixture at $\mathrm{C} 6,7$ )

To a solution of $\mathbf{1 3}(1.99 \mathrm{~g}, 5.56 \mathrm{mmol})$ in benzene $(56 \mathrm{~mL})$ was added $\mathrm{VO}(\mathrm{acac})_{2}(284 \mathrm{mg}, 1.11$ $\mathrm{mmol}$ ) and TBHP (5.0-6.0 $\mathrm{M}$ in decane, $2.2 \mathrm{ml}, 11 \mathrm{mmol})$, and the mixture was stirred for 30 min at room temperature. The reaction mixture was quenched with sat. $\mathrm{Na}_{2} \mathrm{~S}_{2} \mathrm{O}_{3}$ aqueous solution, and the mixture was stirred for $2.5 \mathrm{~h}$ at same temperature. The mixture was extracted with AcOEt (three times). The combined organic layers were washed with brine, dried over $\mathrm{MgSO}_{4}$, and concentrated in vacuo. The resulting residue was purified by silica gel column chromatography (hexane-AcOEt, 40:1) to afford 14 (1.72 g, 83\%) as a mixture of diastereomers $(4: 1$ at $\mathrm{C} 6,7)$ as yellow oil. 
$[\alpha]_{\mathrm{D}}{ }^{25}+113.92\left(c 0.29, \mathrm{CHCl}_{3}\right)$. IR (neat): 3464, 2955, 2929, 2857, 1255, 1114, 1097, 1073, 884, $835,799,774 \mathrm{~cm}^{-1}$. ${ }^{1} \mathrm{H}$ NMR for only major isomer $\left(\mathrm{CDCl}_{3}, 400 \mathrm{MHz}\right): \delta 7.07-7.30(\mathrm{~m}, 4 \mathrm{H})$, $5.66(\mathrm{~m}, 1 \mathrm{H}), 5.51(\mathrm{dd}, 1 \mathrm{H}, J=5.9,1.4 \mathrm{~Hz}), 4.27(\mathrm{t}, 1 \mathrm{H}, J=1.6 \mathrm{~Hz}), 4.05(\mathrm{~d}, 1 \mathrm{H}, J=4.6 \mathrm{~Hz})$, $3.13(\mathrm{~d}, 1 \mathrm{H}, J=4.6 \mathrm{~Hz}), 2.34(\mathrm{~s}, 3 \mathrm{H}), 1.17(\mathrm{~s}, 3 \mathrm{H}), 0.89-0.85(\mathrm{~s}, 12 \mathrm{H}), 0.06(\mathrm{~m}, 6 \mathrm{H})$, peak of -OH does not exist because of exchange to $\mathrm{CDCl}_{3}$ deuterium. ${ }^{13} \mathrm{C}$ NMR for only major isomer $\left(\mathrm{CDCl}_{3}, 400 \mathrm{MHz}\right): \delta 138.1,136.3,135.3,133.3,128.6,128.3,126.6,123.2,83.1,82.1,60.6$, 56.8, 53.3, 25.9, 21.6, 20.7, 18.2, 18.1, -4.4, -4.8. HRMS (ESI-TOF) m/z: $[\mathrm{M}+\mathrm{Na}]^{+}$calcd for $\mathrm{C}_{22} \mathrm{H}_{34} \mathrm{O}_{3} \mathrm{SiNa}$ 397.2175; Found 397.2167.

$(1 R, 3 S)-1-((R)-1-h y d r o x y-2-(m$-tolyl)ethyl)-2,2-dimethylcyclopent-4-ene-1,3-diol (15)

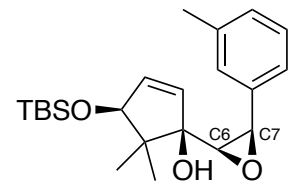

14

(4:1 diastereomer mixture at $\mathrm{C} 6,7)$

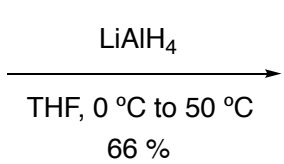

$66 \%$

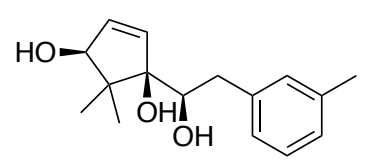

15

(single isomer after column separation)

To a suspension of $\mathrm{LiAlH}_{4}(691 \mathrm{mg}, 18.2 \mathrm{mmol})$ in THF (46 mL) was added dropwise 14 (1.71 $\mathrm{g}, 4.56 \mathrm{mmol})$ in THF $(9 \mathrm{ml})$ at $0{ }^{\circ} \mathrm{C}$ under $\mathrm{Ar}$, and the reaction mixture was stirred for $2 \mathrm{~h}$ at 50 ${ }^{\circ} \mathrm{C}$. After cooling to $0{ }^{\circ} \mathrm{C}$, the reaction mixture was carefully quenched with $\mathrm{H}_{2} \mathrm{O}(0.69 \mathrm{ml}), 15 \%$ $\mathrm{NaOH}$ aqueous solution $(0.69 \mathrm{ml})$ and $\mathrm{H}_{2} \mathrm{O}(2 \mathrm{ml})$ at the same temperature, and the mixture was stirred for $45 \mathrm{~min}$ at room temperature. The insoluble material was filtered and concentrated in vacuo. The resulting residue was purified by silica gel column chromatography (hexane-AcOEt, $3: 1)$ to afford $15(786 \mathrm{mg}, 66 \%)$ as a single diastereomer as colorless oil.

$[\alpha]_{\mathrm{D}}{ }^{25}+64.18\left(c 0.28, \mathrm{CHCl}_{3}\right)$. IR (neat): 3398, 3058, 2958, 1608, 1469, 1384, 1365, 1321, 1262 , 1039, 771, $699 \mathrm{~cm}^{-1} .{ }^{1} \mathrm{H}$ NMR $\left(\mathrm{CDCl}_{3}, 400 \mathrm{MHz}\right): \delta$ 7.26-7.21 (m, 1H), 7.08-7.02 (m, 3H), 6.20 $(\mathrm{dd}, 1 \mathrm{H}, J=5.9,2.3 \mathrm{~Hz}), 6.00(\mathrm{~d}, 1 \mathrm{H}, J=5.9 \mathrm{~Hz}), 4.09$ (d, 1H, $J=2.3 \mathrm{~Hz}), 3.88$ (dd, 1H, $J=$ 10.1, 3.2 Hz), 2.89-2.80 (m, 2H), $2.35(\mathrm{~s}, 3 \mathrm{H}), 1.18(\mathrm{~s}, 3 \mathrm{H}), 1.08(\mathrm{~s}, 3 \mathrm{H})$, peak of -OH does not exist because of exchange to $\mathrm{CDCl}_{3}$ proton. ${ }^{13} \mathrm{C} \mathrm{NMR}\left(\mathrm{CDCl}_{3}, 400 \mathrm{MHz}\right): \delta 138.7,138.0,136.3$, $130.4,128.9,127.8,126.6,86.4,84.4,73.4,49.2,39.0,24.0,21.6,18.5$. HRMS (ESI-TOF) $\mathrm{m} / \mathrm{z}:[\mathrm{M}+\mathrm{Na}]^{+}$calcd for $\mathrm{C}_{16} \mathrm{H}_{22} \mathrm{O}_{3} \mathrm{Na} 285.1467$; Found 285.1461. 


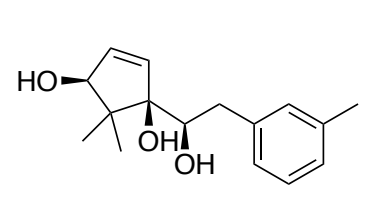

15

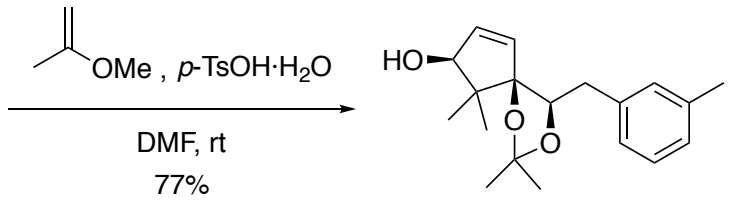

16

To a solution of 15 (701 g, $2.67 \mathrm{mmol})$ in DMF (14 mL) was added 2-methoxypropene $(0.77 \mathrm{ml}$, $8.04 \mathrm{mmol}$ ) and $p$-TsOH (138 $\mathrm{mg}, 0.801 \mathrm{mmol})$, and the mixture was stirred for $21 \mathrm{~h}$ at room temperature. The reaction mixture was quenched with sat. $\mathrm{NaHCO}_{3}$ aqueous solution and extracted with $\mathrm{Et}_{2} \mathrm{O}$ (three times). The combined organic layers were washed with brine, dried over $\mathrm{MgSO}_{4}$, and concentrated in vacuo. The resulting residue was purified by silica gel column chromatography (hexane-AcOEt, 7:1) to afford 16 (619 mg, 77\%) as yellow oil.

$[\alpha]_{\mathrm{D}}{ }^{25}+83.37$ (c 0.29, $\mathrm{CHCl}_{3}$ ). IR (neat): 3426, 2984, 2958, 2934, 1379, 1366, 1254, 1218, 1173 , 1091, 1045, 773, $697 \mathrm{~cm}^{-1} .{ }^{1} \mathrm{H}$ NMR $\left(\mathrm{CDCl}_{3}, 400 \mathrm{MHz}\right): \delta$ 7.26-7.17 (m, 1H), 7.08-7.03 (m, $3 \mathrm{H}), 6.13(\mathrm{~d}, 1 \mathrm{H}, J=5.7 \mathrm{~Hz}), 6.06(\mathrm{~d}, 1 \mathrm{H}, J=5.9 \mathrm{~Hz}), 4.21(\mathrm{dd}, 1 \mathrm{H}, J=9.6,1.8 \mathrm{~Hz}), 4.02(\mathrm{~d}$, $1 \mathrm{H}, J=2.3 \mathrm{~Hz}), 2.79(\mathrm{dd}, 1 \mathrm{H}, J=15.1,9.6 \mathrm{~Hz}), 2.60$ (dd, 1H, $J=14.6,1.4 \mathrm{~Hz}), 2.34(\mathrm{~s}, 3 \mathrm{H})$, $1.44(\mathrm{~s}, 3 \mathrm{H}), 1.30(\mathrm{~s}, 3 \mathrm{H}), 1.10(\mathrm{~s}, 3 \mathrm{H}), 1.05(\mathrm{~s}, 3 \mathrm{H})$, peak of $-\mathrm{OH}$ does not exist because of exchange to $\mathrm{CDCl}_{3}$ deuterium. ${ }^{13} \mathrm{C} \mathrm{NMR}\left(\mathrm{CDCl}_{3}, 400 \mathrm{MHz}\right): \delta 139.2,138.1,136.0,135.4$, 129.7, 128.4, 127.3, 126.0, 107.6, 94.2, 83.4, 78.3, 46.2, 37.3, 29.2, 26.7, 24.5, 21.6, 18.3. HRMS (ESI-TOF) m/z: [M + Na $]^{+}$calcd for $\mathrm{C}_{19} \mathrm{H}_{26} \mathrm{O}_{3} \mathrm{Na} 325.1780$; Found 325.1779.

$N, N$-dimethyl-2-((4R,5R,6R)-2,2,9,9-tetramethyl-4-(3-methylbenzyl)-1,3-dioxaspiro[4.4]non-7en-6-yl)acetamide (17)

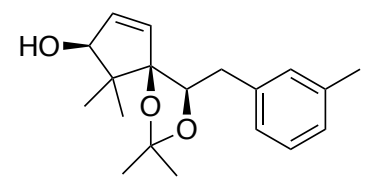

16

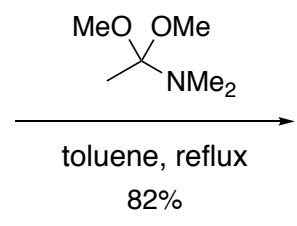

$82 \%$

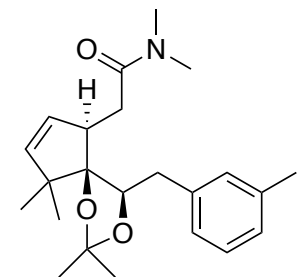

17

To a solution of $16(542 \mathrm{mg}, 1.79 \mathrm{mmol})$ in toluene $(6 \mathrm{~mL})$ was added $N, N$-dimethylacetamide dimethyl acetal (90\% purity, $0.87 \mathrm{ml}, 5.36 \mathrm{mmol})$ under Ar, and the mixture was stirred for 3 days under reflux condition. The reaction mixture was quenched with $\mathrm{H}_{2} \mathrm{O}$ and extracted with AcOEt (twice). The combined organic layers were washed with brine, dried over $\mathrm{MgSO}_{4}$, and concentrated in vacuo. The resulting residue was purified by silica gel column chromatography (hexane-AcOEt, 5:1) to afford 17 (544 g, 82\%) as yellow oil.

$[\alpha]_{\mathrm{D}}^{25}-62.95\left(c 0.24, \mathrm{CHCl}_{3}\right)$. IR (neat): 3452, 2988, 2936, 2869, 1650, 1396, 1378, 1262, 1208 , 
1158, 1092, 1049, $768 \mathrm{~cm}^{-1} .{ }^{1} \mathrm{H}$ NMR ( $\left.\mathrm{CDCl}_{3}, 400 \mathrm{MHz}\right): \delta 7.19$ (m, 1H), 7.10 (m, 2H), 7.03 (d, $1 \mathrm{H}, J=7.3 \mathrm{~Hz}), 5.69(\mathrm{dd}, 1 \mathrm{H}, J=5.9,1.8 \mathrm{~Hz}), 5.59(\mathrm{dd}, 1 \mathrm{H}, J=5.9,2.7 \mathrm{~Hz}), 4.27(\mathrm{dd}, 1 \mathrm{H}, J=$ 10.1, $1.8 \mathrm{~Hz}), 3.50(\mathrm{~m}, 1 \mathrm{H}), 3.02-2.85(\mathrm{~m}, 8 \mathrm{H}), 2.64-2.62(\mathrm{~m}, 2 \mathrm{H}), 2.34(\mathrm{~s}, 3 \mathrm{H}), 1.50(\mathrm{~s}, 3 \mathrm{H})$, $1.33(\mathrm{~s}, 3 \mathrm{H}), 1.16(\mathrm{~s}, 3 \mathrm{H}), 1.07(3 \mathrm{H}, \mathrm{s}) .{ }^{13} \mathrm{C} \mathrm{NMR}\left(\mathrm{CDCl}_{3}, 400 \mathrm{MHz}\right): \delta 172.4,139.5,139.2$, 138.0, 131.0, 129.8, 128.4, 127.2, 125.9, 107.0, 94.7, 78.1, 49.3, 45.0, 37.9, 37.4, 35.6, 34.3, 27.3, 26.4, 24.2, 22.5, 21.6. HRMS (ESI-TOF) m/z: $[\mathrm{M}+\mathrm{Na}]^{+}$calcd for $\mathrm{C}_{23} \mathrm{H}_{33} \mathrm{NO}_{3} \mathrm{Na}$ 394.2358; Found 394.2351.

(3a $R, 6 \mathrm{a} R)-6 \mathrm{a}-((R)-1$-hydroxy-2-( $m$-tolyl)ethyl)-6,6-dimethyl-3,3a,6,6a-tetrahydro-2H-cyclopent $\mathrm{a}[b]$ furan-2-one (S2)

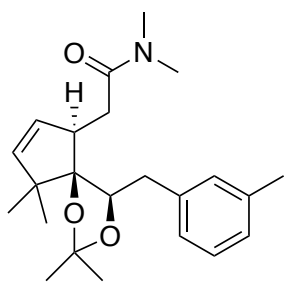

17

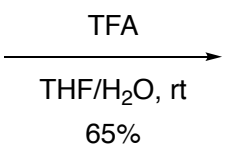

$65 \%$

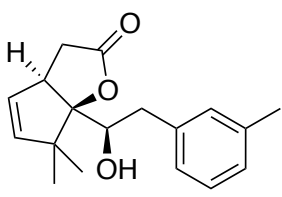

S2

To a solution of $17(526 \mathrm{mg}, 1.42 \mathrm{mmol})$ in THF/ $\mathrm{H}_{2} \mathrm{O}(4 \mathrm{~mL} / 1 \mathrm{~mL})$ was added TFA (4 ml), and the mixture was stirred for $5 \mathrm{~h}$ at $50{ }^{\circ} \mathrm{C}$. The reaction mixture was quenched with sat. $\mathrm{NaHCO}_{3}$ aqueous solution and extracted with AcOEt (twice). The combined organic layers were washed with brine, dried over $\mathrm{MgSO}_{4}$, and concentrated in vacuo. The resulting residue was purified by silica gel column chromatography $\left(\mathrm{CHCl}_{3}\right.$-AcOEt, 50:1) to afford $\mathbf{S 2}(264 \mathrm{mg}, 65 \%)$ as colorless oil.

$[\alpha]_{\mathrm{D}}{ }^{25}+12.95$ (c 0.23, $\left.\mathrm{CHCl}_{3}\right)$. IR (neat): 3467, 2939, 1770, 1297, 1206, 1173, 1095, 1052, 1025, 1000, 979, 775, $753 \mathrm{~cm}^{-1} .{ }^{1} \mathrm{H} \mathrm{NMR}\left(\mathrm{CDCl}_{3}, 400 \mathrm{MHz}\right): \delta 7.26-7.21(\mathrm{~m}, 1 \mathrm{H}), 7.09-7.00(\mathrm{~m}, 3 \mathrm{H})$, $5.58(\mathrm{dd}, 1 \mathrm{H}, J=5.9,1.8 \mathrm{~Hz}), 5.34(\mathrm{dd}, 1 \mathrm{H}, J=5.7,1.6 \mathrm{~Hz}), 4.13(\mathrm{~m}, 1 \mathrm{H}), 3.41(\mathrm{~m}, 1 \mathrm{H})$, 2.89-2.79 (m, 2H), 2.64 (dd, 1H, $J=13.0,11.2 \mathrm{~Hz}), 2.45$ (d, 1H, $J=18.3 \mathrm{~Hz}), 2.34(\mathrm{~s}, 3 \mathrm{H}), 1.30$ $(\mathrm{s}, 3 \mathrm{H}), 1.23(\mathrm{~s}, 3 \mathrm{H})$, peak of $-\mathrm{OH}$ does not exist because of exchange to $\mathrm{CDCl}_{3}$ deuterium. ${ }^{13} \mathrm{C}$ NMR (CDCl $3,400 \mathrm{MHz}): \delta 176.4,142.9,138.6,137.1,130.2,128.9,127.9,126.5,126.4,98.4$, 75.0, 52.3, 46.9, 38.9, 35.1, 26.2, 23.3, 21.6. HRMS (ESI-TOF) $\mathrm{m} / \mathrm{z}:[\mathrm{M}+\mathrm{Na}]^{+}$calcd for $\mathrm{C}_{18} \mathrm{H}_{22} \mathrm{O}_{3} \mathrm{Na}$ 309.1467; Found 309.1460.

(3a $R, 6 \mathrm{a} R)-6 \mathrm{a}-((R)-1-($ methoxymethoxy)-2-( $m$-tolyl)ethyl)-6,6-dimethyl-3,3a,6,6a-tetrahydro-2H -cyclopenta[b]furan-2-one (18) 


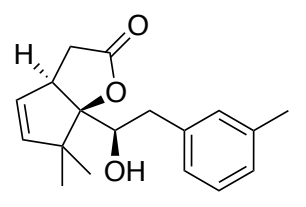

S2

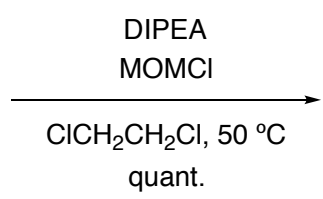

quant.

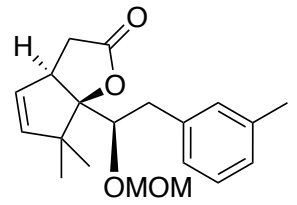

18

To a solution of $\mathbf{S 2}(218 \mathrm{mg}, 0.761 \mathrm{mmol})$ in $\mathrm{ClCH}_{2} \mathrm{CH}_{2} \mathrm{Cl}(3.8 \mathrm{~mL})$ was added DIPEA $(0.8 \mathrm{ml}$, $4.59 \mathrm{mmol}) \mathrm{MOMCl}(0.35 \mathrm{ml}, 4.64 \mathrm{mmol})$ at $0{ }^{\circ} \mathrm{C}$, and the mixture was stirred for $13 \mathrm{~h}$ at $50{ }^{\circ} \mathrm{C}$. The reaction mixture was quenched with sat. $\mathrm{NaHCO}_{3}$ aqueous solution and extracted with $\mathrm{CH}_{2} \mathrm{Cl}_{2}$ (twice). The combined organic layers were washed with brine, dried over $\mathrm{MgSO}_{4}$, and concentrated in vacuo. The resulting residue was purified by silica gel column chromatography (hexane-AcOEt, 5:1) to afford $\mathbf{1 8}$ (251 mg, quant.) as colorless oil.

$[\alpha]_{\mathrm{D}}{ }^{25}-57.46\left(c 0.20, \mathrm{CHCl}_{3}\right)$. IR (neat): 2964, 2937, 1775, 1205, 1172, 1152, 1096, 1073, 1037 , 1018, 974, $755 \mathrm{~cm}^{-1} .{ }^{1} \mathrm{H}$ NMR $\left(\mathrm{CDCl}_{3}, 400 \mathrm{MHz}\right): \delta 7.18$ (t, 1H, $\left.J=7.5 \mathrm{~Hz}\right), 7.06-7.03(\mathrm{~m}, 3 \mathrm{H})$, $5.55(\mathrm{dd}, 1 \mathrm{H}, J=5.7,2.1 \mathrm{~Hz}), 5.38(\mathrm{dd}, 1 \mathrm{H}, J=5.9,1.8 \mathrm{~Hz}), 4.33(\mathrm{~d}, 1 \mathrm{H}, J=6.9 \mathrm{~Hz}), 4.01(\mathrm{~d}$, $1 \mathrm{H}, J=6.9 \mathrm{~Hz}), 3.92(\mathrm{dd}, 1 \mathrm{H}, J=10.3,2.1 \mathrm{~Hz}), 3.64(\mathrm{dd}, 1 \mathrm{H}, J=8.7,1.8 \mathrm{~Hz}), 3.14-3.09$ (m, $4 \mathrm{H}), 2.87-2.81(\mathrm{~m}, 2 \mathrm{H}), 2.42(\mathrm{~m}, 1 \mathrm{H}), 2.33(\mathrm{~s}, 3 \mathrm{H}), 1.30(\mathrm{~m}, 6 \mathrm{H}) .{ }^{13} \mathrm{C} \mathrm{NMR}\left(\mathrm{CDCl}_{3}, 400 \mathrm{MHz}\right)$ : $\delta 176.3,142.7,138.7,138.2,130.1,128.5,127.4,126.9,126.4,98.5,97.9,81.8,56.2,51.3,46.5$, 38.5, 34.5, 25.8, 23.6, 21.5. HRMS (ESI-TOF) $\mathrm{m} / \mathrm{z}$ : $[\mathrm{M}+\mathrm{Na}]^{+}$calcd for $\mathrm{C}_{20} \mathrm{H}_{26} \mathrm{O}_{4} \mathrm{Na} 353.1729$; Found 353.1721 .

(3S,3aS,6aR)-6a-((R)-1-(methoxymethoxy)-2-(m-tolyl)ethyl)-3,6,6-trimethyl-3,3a,6,6a-tetrahydr o- $2 H$-cyclopenta[b]furan-2-one (19)

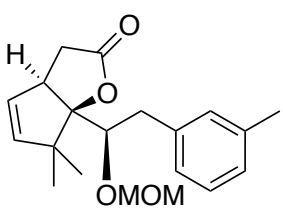

18

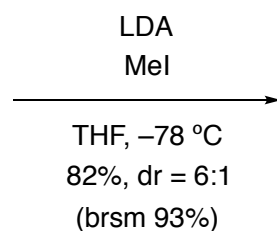

(brsm 93\%)

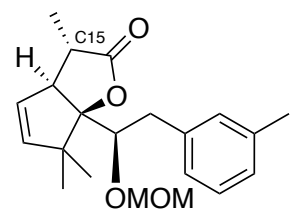

19

(diastereomer mixutre at C15)

To a stirred solution $i \operatorname{Pr}_{2} \mathrm{NH}(0.123 \mathrm{~mL}, 0.870 \mathrm{mmol})$ in THF $(1.6 \mathrm{~mL})$ was added dropwise $n$-BuLi (1.49 $\mathrm{M}$ in $n$-hexane, $0.541 \mathrm{~mL}, 0.806 \mathrm{mmol})$ at $-78{ }^{\circ} \mathrm{C}$ under $\mathrm{Ar}$, and the mixture was stirred for $30 \mathrm{~min}$ at $0{ }^{\circ} \mathrm{C}$. After cooling to $-78{ }^{\circ} \mathrm{C}$, to the mixture was added dropwise a solution of $18(213 \mathrm{mg}, 0.645 \mathrm{mmol})$ in $\operatorname{THF}(1.3 \mathrm{~mL})$ at $-78^{\circ} \mathrm{C}$, and the mixture was stirred for $1 \mathrm{~h}$ at the same temperature. To the mixture was added dropwise MeI $(0.0442 \mathrm{ml}, 0.709 \mathrm{mmol})$ at -78 ${ }^{\circ} \mathrm{C}$, and the mixture was stirred for $7 \mathrm{~h}$ at the same temperature. The reaction mixture was quenched with sat. $\mathrm{NH}_{4} \mathrm{Cl}$ aqueous solution, and extracted with AcOEt (twice). The combined 
organic layers were washed with brine, dried over $\mathrm{MgSO}_{4}$, and concentrated in vacuo. The resulting residue was purified by silica gel column chromatography (hexane-AcOEt, 10:1) to afford 19 (183 mg, 83\%, brsm 93\%) as a mixture of diastereomers (6:1 at C13) and yellow oil. $[\alpha]_{\mathrm{D}}{ }^{25}+5.77\left(c 0.21, \mathrm{CHCl}_{3}\right)$. IR (neat): 2968, 2937, 1772, 1461, 1205, 1152, 1097, 1045, 1028 , 975, 921, 754, $698 \mathrm{~cm}^{-1} .{ }^{1} \mathrm{H}$ NMR for only major isomer $\left(\mathrm{C}_{6} \mathrm{D}_{6}, 400 \mathrm{MHz}\right): \delta 6.90-7.13(\mathrm{~m}, 4 \mathrm{H})$, $5.20(\mathrm{~m}, 1 \mathrm{H}), 5.11(\mathrm{dd}, 1 \mathrm{H}, J=5.7,2.1 \mathrm{~Hz}), 4.50(\mathrm{~d}, 1 \mathrm{H}, J=5.5 \mathrm{~Hz}), 4.18(\mathrm{~d}, 1 \mathrm{H}, J=6.4 \mathrm{~Hz})$, 4.07 (d, 1H, $J=9.6 \mathrm{~Hz}), 3.16(\mathrm{~m}, 1 \mathrm{H}), 3.03$ (m, 1H), 2.84 (s, 3H), 2.69-2.62 (m, 1H), 2.23 (qd, $1 \mathrm{H}, J=7.6,4.1 \mathrm{~Hz}), 2.14(\mathrm{~s}, 3 \mathrm{H}), 1.30(\mathrm{~d}, 3 \mathrm{H}, J=7.3), 1.11(\mathrm{~s}, 3 \mathrm{H}), 1.09(\mathrm{~s}, 3 \mathrm{H}) .{ }^{13} \mathrm{C}$ NMR for only major isomer $\left(\mathrm{CDCl}_{3}, 400 \mathrm{MHz}\right): \delta 179.7,142.2,138.8,138.2,130.1,128.5,128.0,127.3$, 126.3, 98.2, 96.0, 55.9, 53.3, 50.7, 42.2, 38.6, 26.4, 24.5, 21.5, 16.8, 11.8. HRMS (ESI-TOF) $\mathrm{m} / \mathrm{z}:[\mathrm{M}+\mathrm{Na}]^{+}$calcd for $\mathrm{C}_{21} \mathrm{H}_{28} \mathrm{O}_{4} \mathrm{Na} 367.1885$; Found 367.1878.

$(1 \mathrm{a} R, 2 \mathrm{a} R, 5 S, 5 \mathrm{a} R, 5 \mathrm{~b} S)-2 \mathrm{a}-((R)-1-(m e t h o x y m e t h o x y)-2-(m$-tolyl)ethyl)-2,2,5-trimethylhexahydro $-4 H$-oxireno[2',3':3,4]cyclopenta[1,2-b]furan-4-one (20)

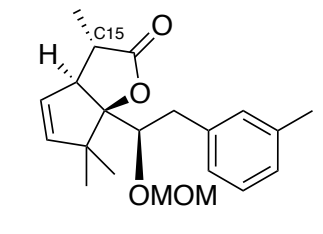

19

(6:1 diastereomer mixutre at $\mathrm{C15})$

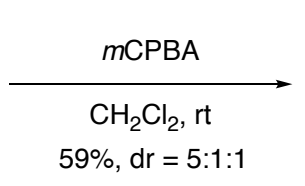

(major isomer after HPLC separation)

To a solution of 19 (6:1 diastereomer mixture, $305 \mathrm{mg}, 0.886 \mathrm{mmol}$ ) in $\mathrm{CH}_{2} \mathrm{Cl}_{2}(3 \mathrm{~mL})$ was added dropwise a solution of $m \mathrm{CPBA}(423 \mathrm{mg}, 1.59 \mathrm{mmol})$ in $\mathrm{CH}_{2} \mathrm{Cl}_{2}(7 \mathrm{~mL})$ at $0{ }^{\circ} \mathrm{C}$, and the mixture was stirred for $22 \mathrm{~h}$ at room temperature. The reaction mixture was quenched with sat. $\mathrm{NaHCO}_{3}$ aqueous solution and extracted with AcOEt (three times). The combined organic layers were washed with brine, dried over $\mathrm{MgSO}_{4}$, and concentrated in vacuo. The resulting residue was purified by silica gel column chromatography (hexane-AcOEt, 7:1) to afford a mixture of diastereomers $(5: 1: 1)$ as colorless oil. Separation of diastereomers by HPLC (hexane-AcOEt, 5:4) afforded $\mathbf{2 0}$ (189 mg, 59\%) as a single diastereomer as colorless oil. $[\alpha]_{\mathrm{D}}{ }^{25}-77.74\left(c 0.26, \mathrm{CHCl}_{3}\right)$. IR (neat): 2972, 2940, 1772, 1211, 1152, 1101, 1075, 1049, 1027 , 1013, 976, $779 \mathrm{~cm}^{-1} .{ }^{1} \mathrm{H}$ NMR ( $\left.\mathrm{CDCl}_{3}, 400 \mathrm{MHz}\right): \delta 7.17$ (t, $\left.1 \mathrm{H}, J=7.5 \mathrm{~Hz}\right), 7.04-7.00(\mathrm{~m}, 3 \mathrm{H})$, $4.24(\mathrm{~d}, 1 \mathrm{H}, J=6.9 \mathrm{~Hz}), 3.81(\mathrm{~d}, 1 \mathrm{H}, J=6.9 \mathrm{~Hz}), 3.62$ (t, 1H, $J=2.3 \mathrm{~Hz}), 3.57$ (d, 1H, $J=10.1$ $\mathrm{Hz}), 3.25(\mathrm{~d}, 1 \mathrm{H}, J=2.7 \mathrm{~Hz}), 3.18-3.15(\mathrm{~m}, 4 \mathrm{H}), 2.98-2.92(\mathrm{~m}, 2 \mathrm{H}), 2.79(\mathrm{~m}, 1 \mathrm{H}), 2.32(\mathrm{~s}, 3 \mathrm{H})$, $1.43(\mathrm{~d}, 3 \mathrm{H}, J=7.8 \mathrm{~Hz}), 1.36$ (s, 3H), 1.32 (s, 3H). ${ }^{13} \mathrm{C} \mathrm{NMR}\left(\mathrm{CDCl}_{3}, 400 \mathrm{MHz}\right): \delta 179.7,138.8$, $138.3,130.2,128.6,127.5,126.4,98.8,95.1,77.4,69.4,59.3,55.9,47.4,44.7,38.7,38.2,21.9$, 
21.5, 21.2, 17.1. HRMS (ESI-TOF) $\mathrm{m} / \mathrm{z}$ : $[\mathrm{M}+\mathrm{Na}]^{+}$calcd for $\mathrm{C}_{21} \mathrm{H}_{28} \mathrm{O}_{5} \mathrm{Na}$ 383.1834; Found 383.1826 .

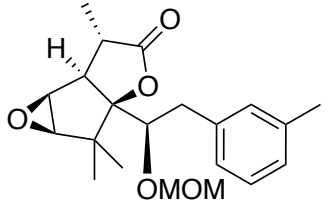

20

(major isomer) (confirmed by $X$-ray)

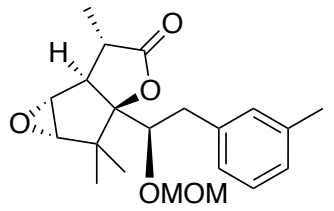

20'

(not confirmed, prediction)

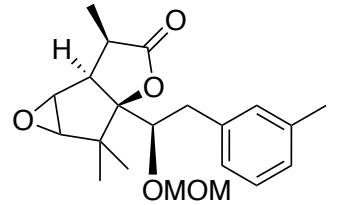

20"

(not confirmed, stereochemistry of epoxide is single but unknown)

20:20':20" = 5:1:1

$(3 S, 3 \mathrm{a} R, 4 R, 5 R, 6 \mathrm{a} R)-4,5$-dihydroxy-6a-(( $R)-1$-hydroxy-2-( $m$-tolyl)ethyl)-3,6,6-trimethylhexahyd ro-2H-cyclopenta[b]furan-2-one (21)

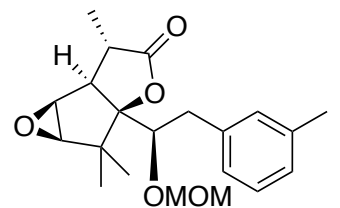

20

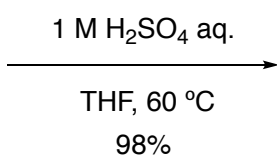

$98 \%$

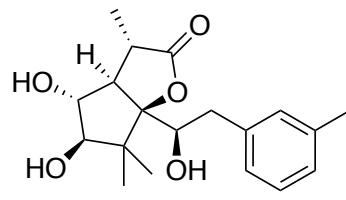

21

To a solution of $20(170 \mathrm{mg}, 0.472 \mathrm{mmol})$ in THF $(4.5 \mathrm{~mL})$ was added $1 \mathrm{M} \mathrm{H}_{2} \mathrm{SO}_{4}$ aqueous solution $(1.5 \mathrm{ml})$, and the mixture was stirred for $34 \mathrm{~h}$ at $60{ }^{\circ} \mathrm{C}$. The reaction mixture was quenched with sat. $\mathrm{NaHCO}_{3}$ aqueous solution and extracted with AcOEt (three times). The combined organic layers were washed with brine, dried over $\mathrm{MgSO}_{4}$, and concentrated in vacuo. The resulting residue was purified by silica gel column chromatography $\left(\mathrm{CHCl}_{3}-\mathrm{AcOEt}, 2: 1\right)$ to afford 21 (155 mg, 98\%) as white solid. The solid was formed by concentration with $\mathrm{CHCl}_{3}$ in vacuo.

Mp 75-80 ${ }^{\circ} \mathrm{C} .[\alpha]_{\mathrm{D}}{ }^{25}+52.95\left(c 0.23, \mathrm{CHCl}_{3}\right)$. IR (KBr): 3412, 2979, 2937, 1746, 1457, 1288, 1251, 1223, 1092, 1047, 1027, 1012, 986, $756 \mathrm{~cm}^{-1} .{ }^{1} \mathrm{H}$ NMR $\left(\mathrm{CDCl}_{3}, 400 \mathrm{MHz}\right): \delta 7.17$ (m, 1H), 7.08-7.04 (m, 3H), 4.06-4.00 (m, 2H), 3.78 (d, 1H, $J=5.5 \mathrm{~Hz}), 2.93(\mathrm{~m}, 1 \mathrm{H}), 2.76(\mathrm{~m}, 1 \mathrm{H})$, $2.61(\mathrm{dd}, 1 \mathrm{H}, J=13.7,11.0 \mathrm{~Hz}), 2.44(\mathrm{t}, 1 \mathrm{H}, J=3.7 \mathrm{~Hz}), 2.35$ (s, 3H), 1.44 (d, 3H, $J=7.3 \mathrm{~Hz})$, $1.34(\mathrm{~s}, 3 \mathrm{H}), 1.07(\mathrm{~s}, 3 \mathrm{H})$, peak of $-\mathrm{OH}$ does not exist because of exchange to $\mathrm{CDCl}_{3}$ deuterium. ${ }^{13} \mathrm{C} \mathrm{NMR}\left(\mathrm{CDCl}_{3}, 400 \mathrm{MHz}\right): \delta 180.3,138.6,137.6,130.2,128.8,127.8,126.4,95.1,85.0,83.2$, 75.5, 52.8, 47.4, 43.3, 38.5, 22.0, 21.6, 20.0, 17.6. HRMS (ESI-TOF) $\mathrm{m} / \mathrm{z}:[\mathrm{M}+\mathrm{Na}]^{+}$calcd for $\mathrm{C}_{19} \mathrm{H}_{26} \mathrm{O}_{5} \mathrm{Na} 357.1678$; Found 357.1670.

$(1 R, 3 R, 4 S, 4 \mathrm{a} R, 5 R, 6 R, 7 \mathrm{a} R)-3,4,7,7-$ tetramethyl-1-(3-methylbenzyl)hexahydro-1 $H$-3,7a-epoxycy clopenta[c]pyran-5,6-diol--1 $1 \lambda^{3}, 13 \lambda^{1}$-trideca-1,3,5,7,9,11-hexayne (1/1) (1) 


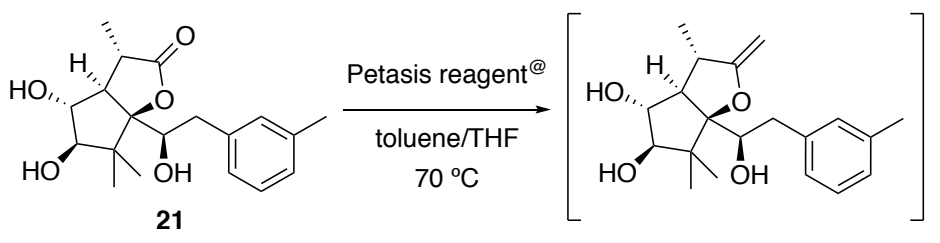

21

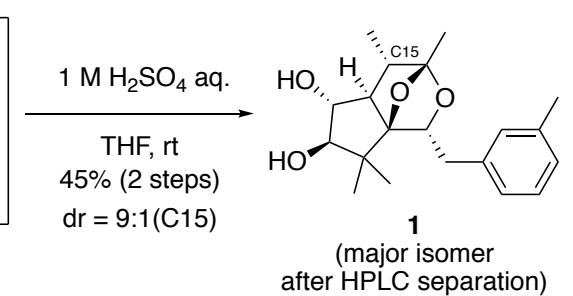

after HPLC separation)

To a solution of Petasis reagent ${ }^{\circledR}(9.15 \%$ in toluene/THF, $4.09 \mathrm{~g}, 1.80 \mathrm{mmol})$ was added a solution of $21(50 \mathrm{mg}, 0.150 \mathrm{mmol})$ in THF $(0.7 \mathrm{ml}) /$ toluene $(0.6 \mathrm{ml})$, and the mixture was stirred for $5 \mathrm{~h}$ at $70^{\circ} \mathrm{C}$ in the dark. After cooling to room temperature, to the reaction mixture was added $\mathrm{NaHCO}_{3}(126 \mathrm{mg}), \mathrm{MeOH}(1.9 \mathrm{ml})$ and $\mathrm{H}_{2} \mathrm{O}(0.07 \mathrm{ml})$, and the mixture was heated at $40{ }^{\circ} \mathrm{C}$ for $18 \mathrm{~h}$. After diluted with $\mathrm{Et}_{2} \mathrm{O}$, the insoluble material was filtered and concentrated in vacuo. To the solution of crude product in THF $(2.2 \mathrm{~mL})$ was added $1 \mathrm{M} \mathrm{H}_{2} \mathrm{SO}_{4}$ aqueous solution $(0.88 \mathrm{ml})$, and the mixture was stirred for $8 \mathrm{~min}$ at room temperature. The reaction mixture was quenched with sat. $\mathrm{NaHCO}_{3}$ aqueous solution and extracted with AcOEt (three times). The combined organic layers were washed with brine, dried over $\mathrm{MgSO}_{4}$, and concentrated in vacuo. The resulting residue was purified by silica gel column chromatography (hexane-AcOEt, 2:1) to afford a mixture of diastereomers (9:1 at C15) and yellow oil. Separation of diastereomers by HPLC (AcOEt) afforded $1(22.5 \mathrm{mg}, 45 \%)$ as a single diastereomer as yellow oil.

$[\alpha]_{\mathrm{D}}{ }^{25}+26.1(c 0.09, \mathrm{MeOH})$. IR (neat): 3409, 2964, 2935, 1395, 1183, 1102, 1066, 998, 873, 822, 784, 756, 705 $\mathrm{cm}^{-1} .{ }^{1} \mathrm{H}$ NMR (CD $\left.\mathrm{CDD}_{3} \mathrm{OD}, 4 \mathrm{MHz}\right): \delta 7.15$ (m, 1H), 7.08 (br, 1H, s), 7.04 (br, d, $1 \mathrm{H}, J=7.6), 7.01$ (br, d, 1H, $J=7.3), 3.93$ (dd, 1H, $J=10.1,1.8 \mathrm{~Hz}), 3.72$ (m, 1H), 3.50 (d, $1 \mathrm{H}, J=6.9 \mathrm{~Hz}), 2.96(\mathrm{dd}, 1 \mathrm{H}, J=14.2,0.9 \mathrm{~Hz}), 2.82(\mathrm{dd}, 1 \mathrm{H}, J=14.2,10.5 \mathrm{~Hz}), 2.31(\mathrm{~s}, 3 \mathrm{H})$, 2.10 (qd, 1H, $J=7.0,3.7 \mathrm{~Hz}), 2.00$ (dd, $1 \mathrm{H}, J=7.8,3.7 \mathrm{~Hz}), 1.36$ (s, 3H), 1.24 (d, 3H, $J=7.3$ $\mathrm{Hz}), 1.20(\mathrm{~s}, 3 \mathrm{H}), 1.07$ (3H, s). ${ }^{13} \mathrm{C}$ NMR (CD $\left.\mathrm{OD}, 400 \mathrm{MHz}\right): \delta 140.7,139.1,130.8,129.4$, $128.1,127.2$, 111.1, 99.1, 89.0, 84.2, 82.0, 52.9, 52.2, 41.4, 38.9, 26.3, 21.6, 19.3, 17.4, 14.5. HRMS (ESI-TOF) m/z: [M + Na $]^{+}$calcd for $\mathrm{C}_{20} \mathrm{H}_{29} \mathrm{O}_{4} 333.2066$; Found 333.2060. 


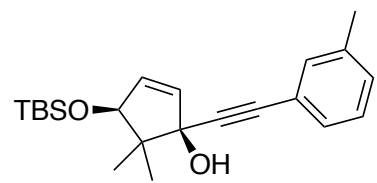

12
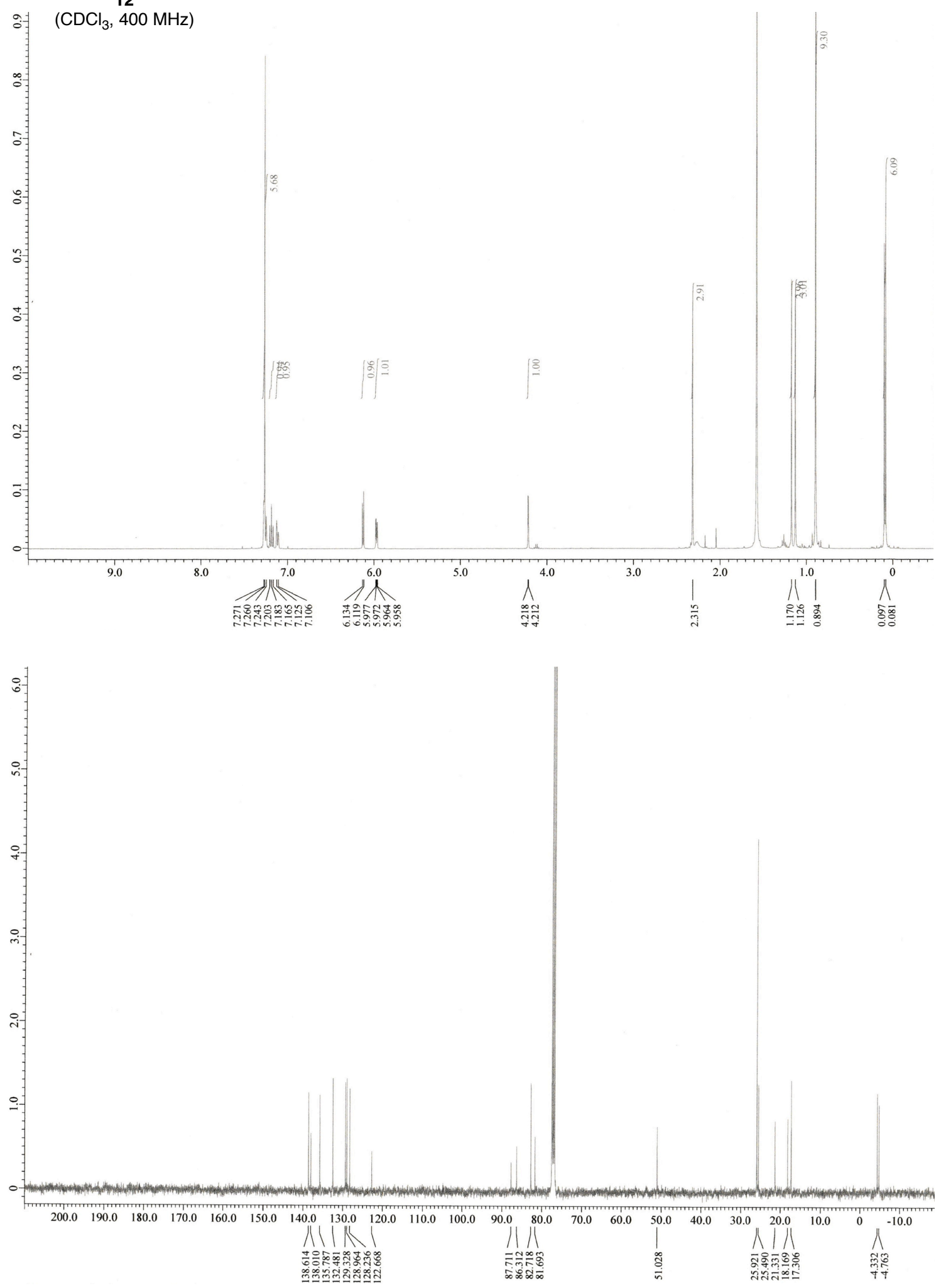


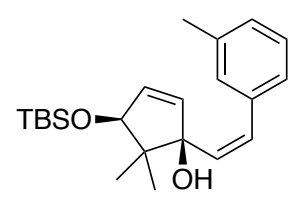

13

$-\left(\mathrm{CDCl}_{3}, 400 \mathrm{MHz}\right)$
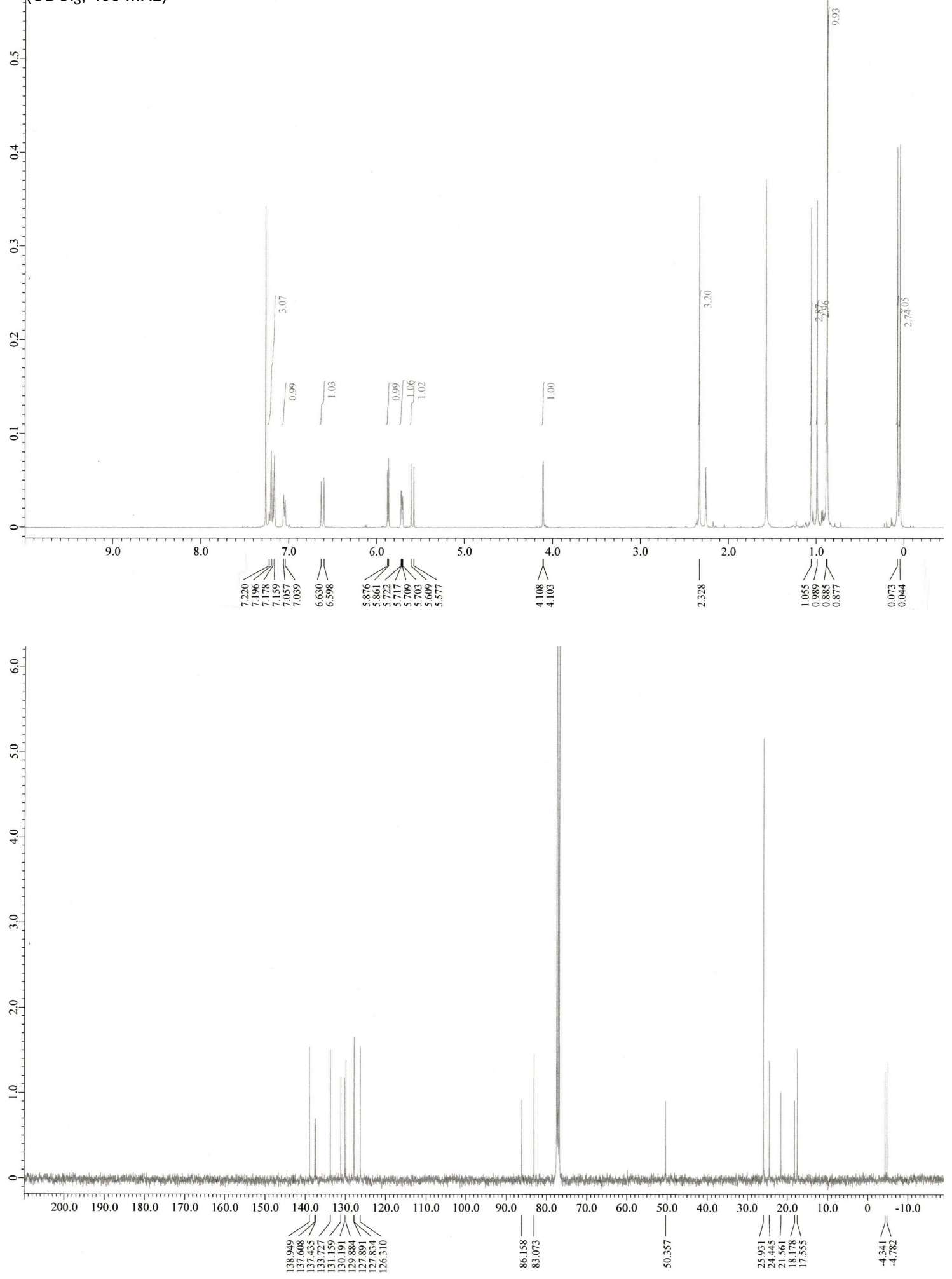


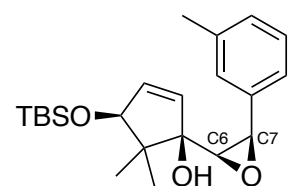

14

(diastereomer mixture at $\mathrm{C} 6,7$ )
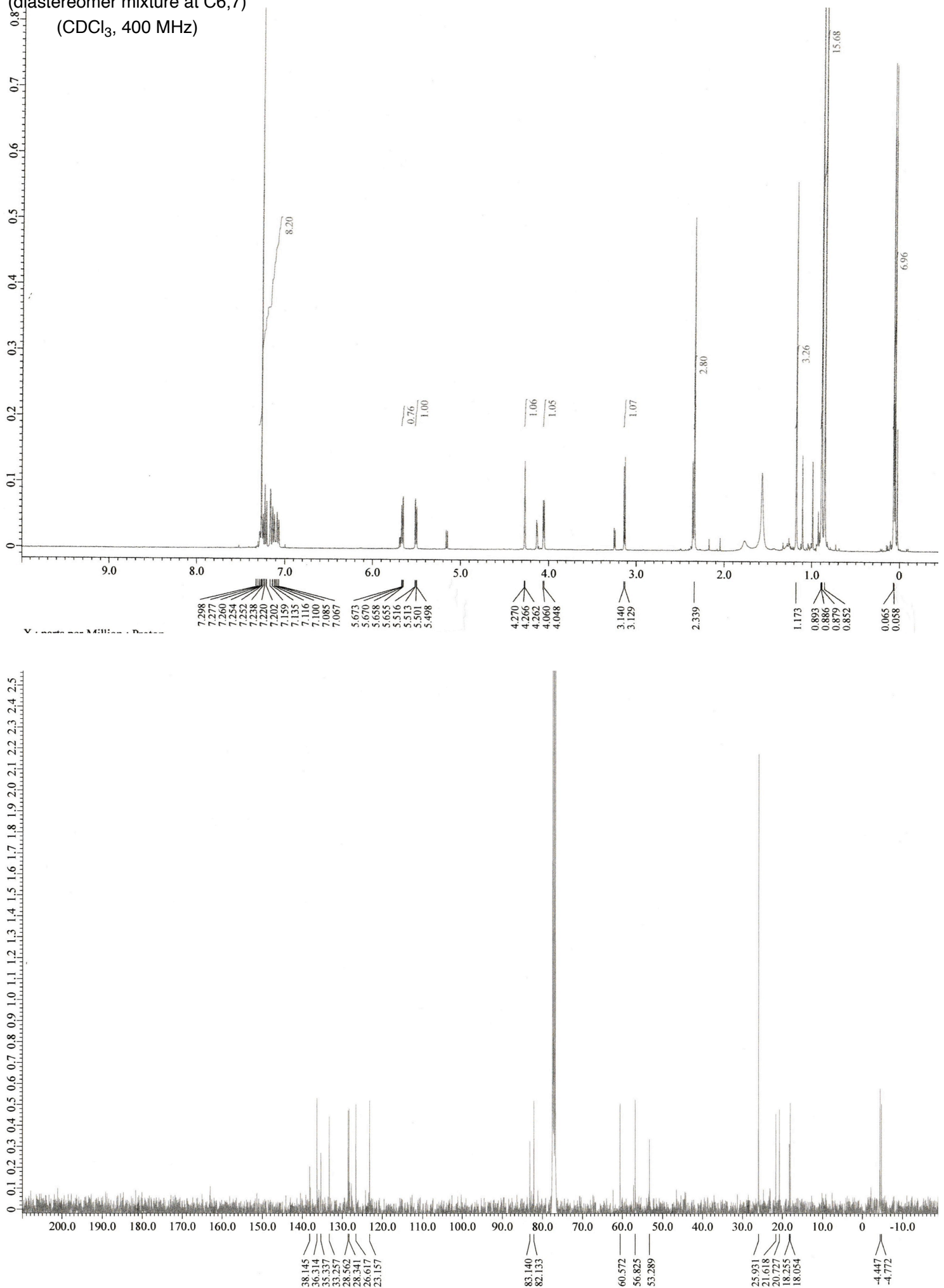


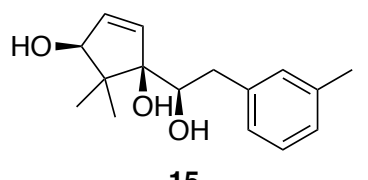

$\left(\mathrm{CDCl}_{3}, 400 \mathrm{MHz}\right)$
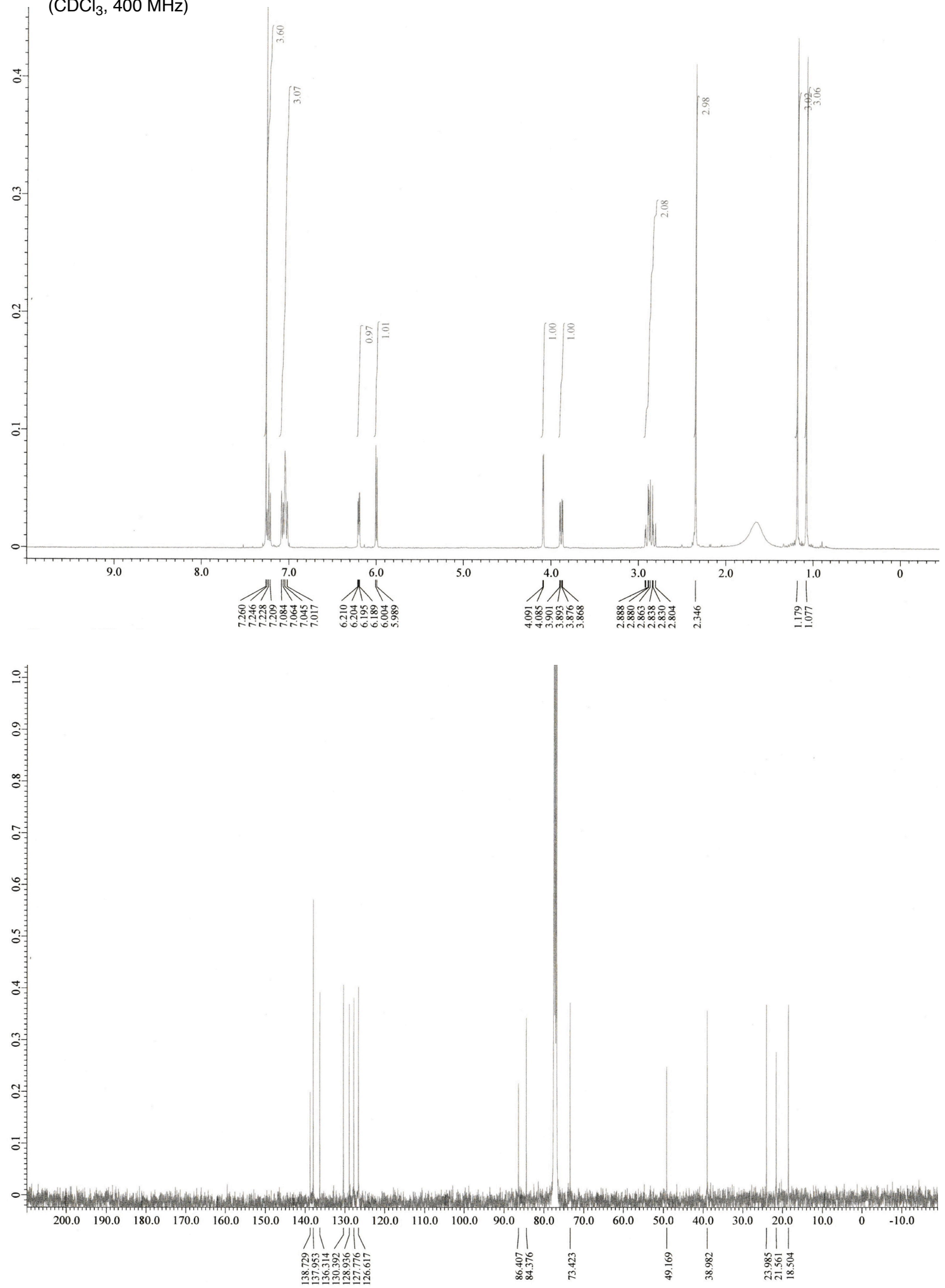

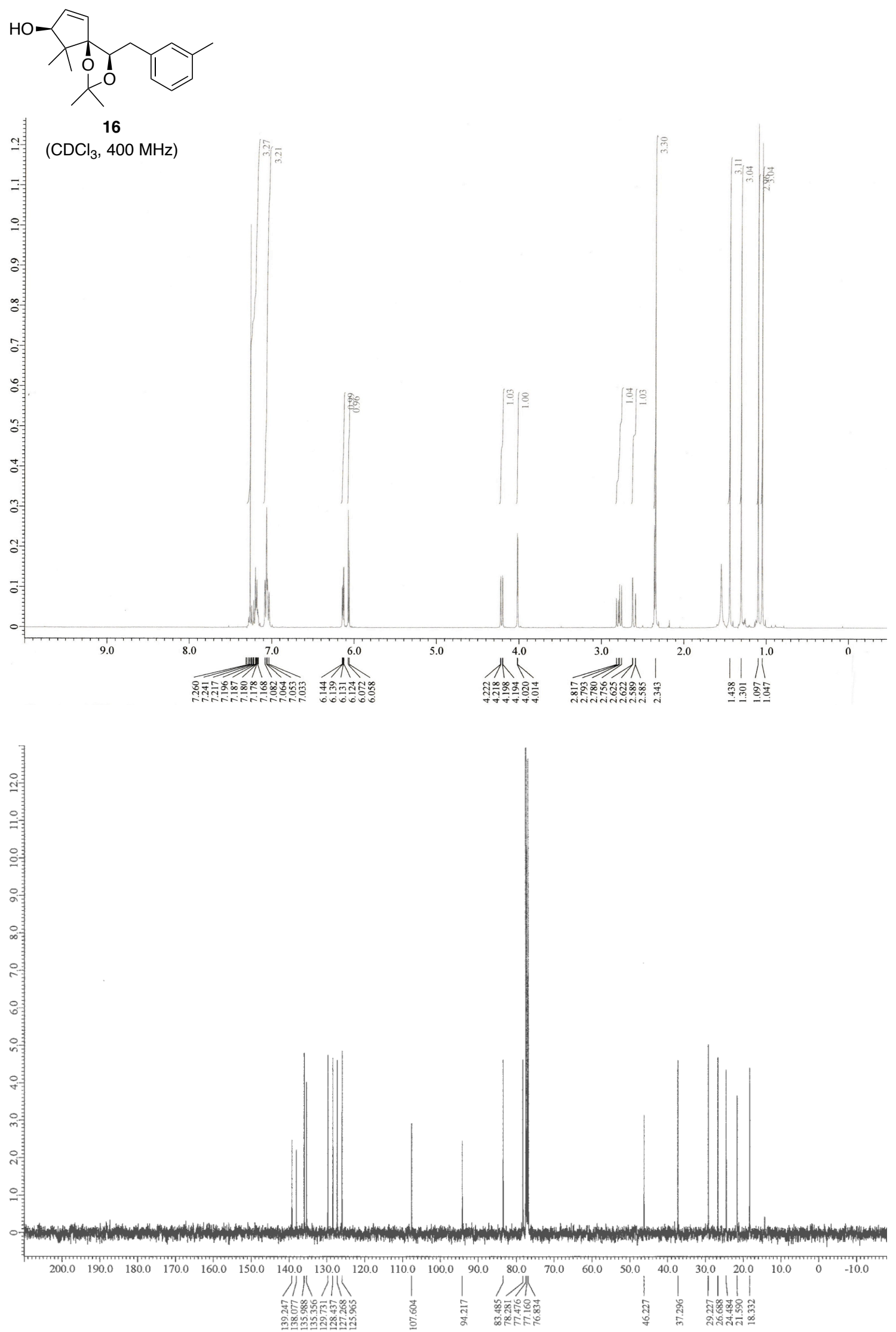

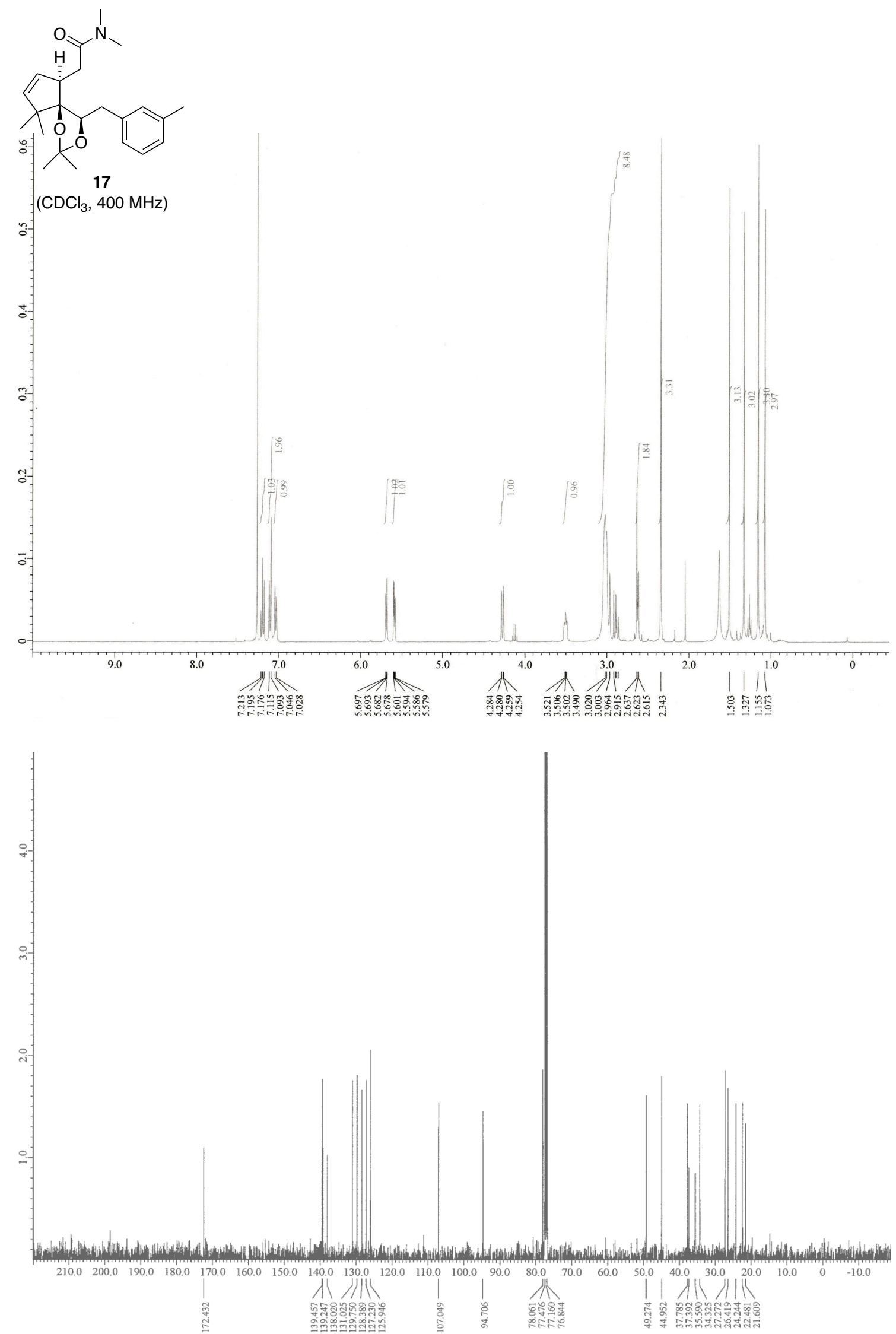


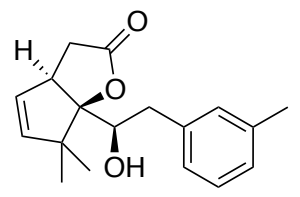

S2

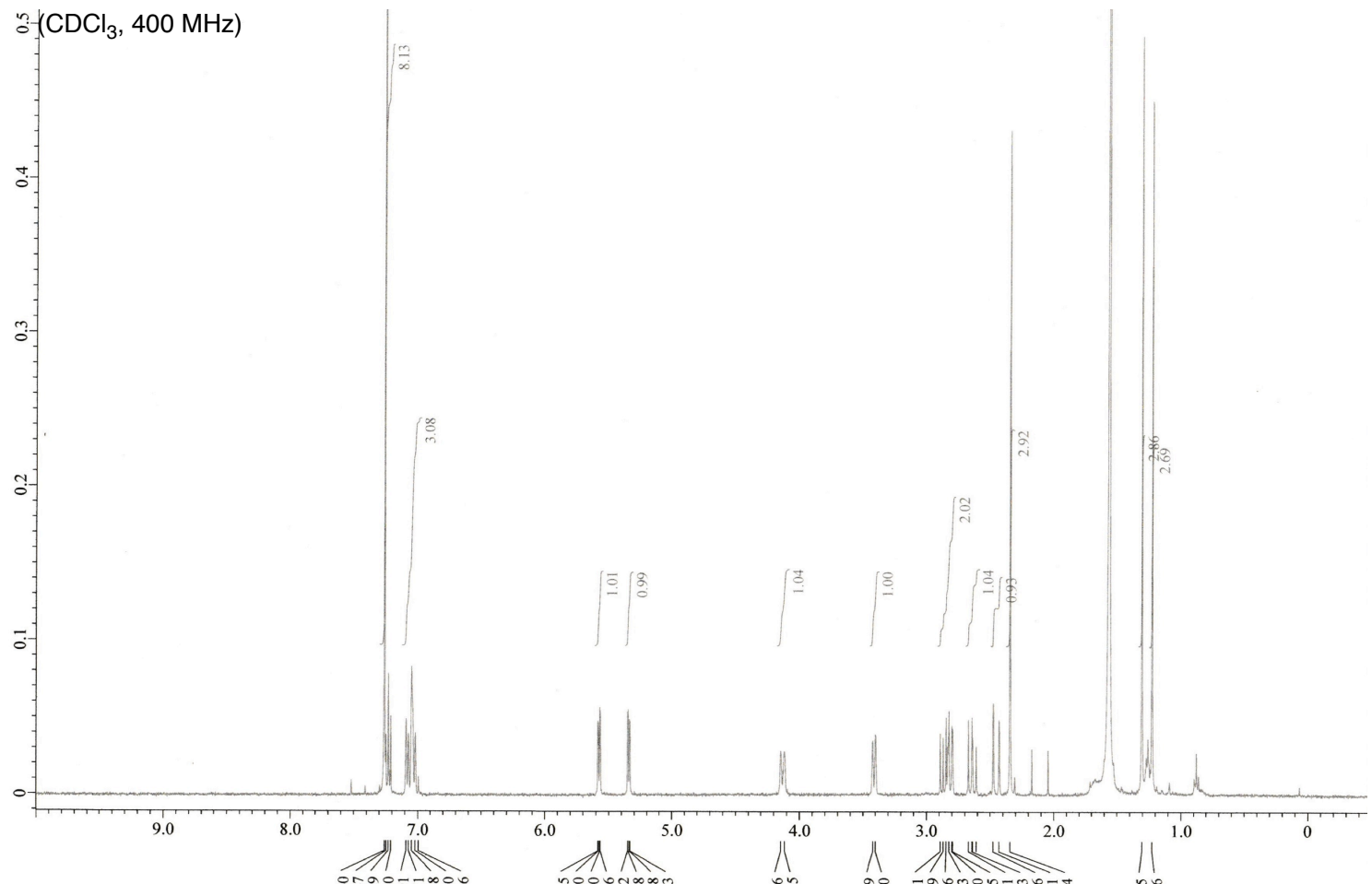

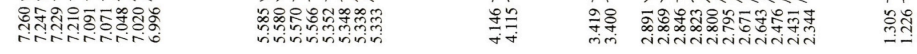

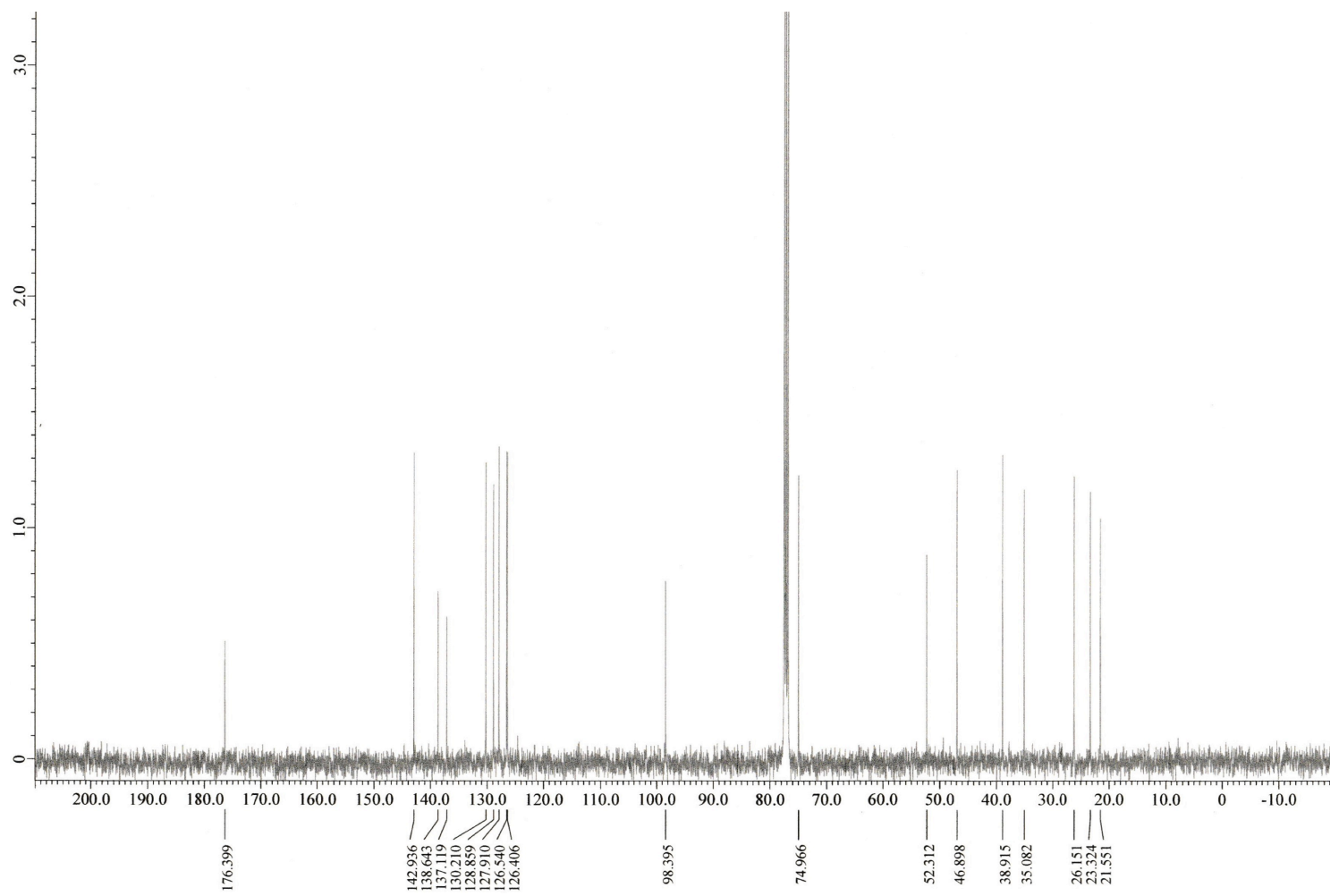



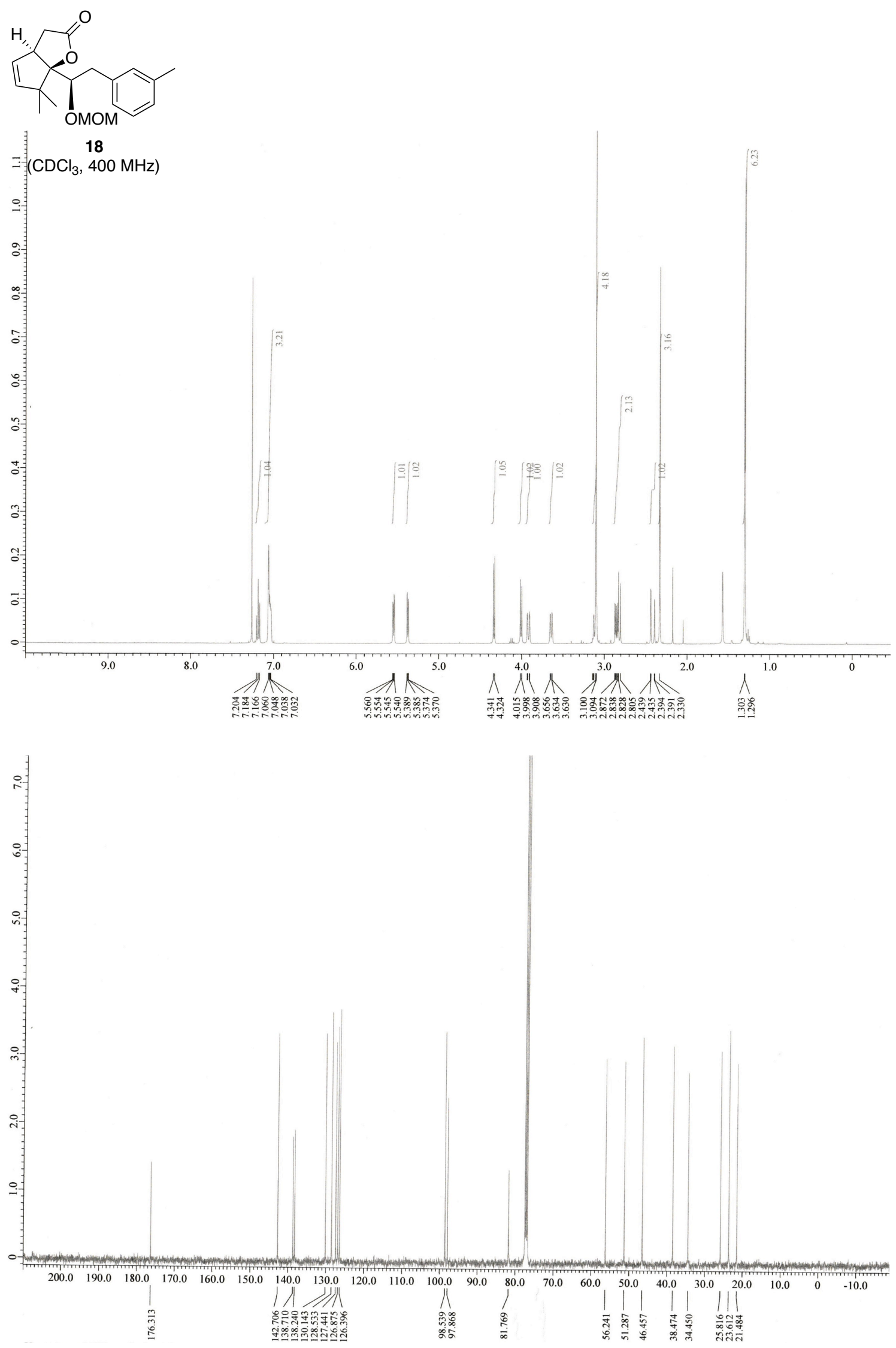


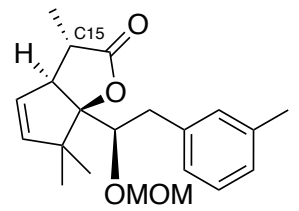

(diastereomer mixutre at $\mathrm{C} 15$ )

-
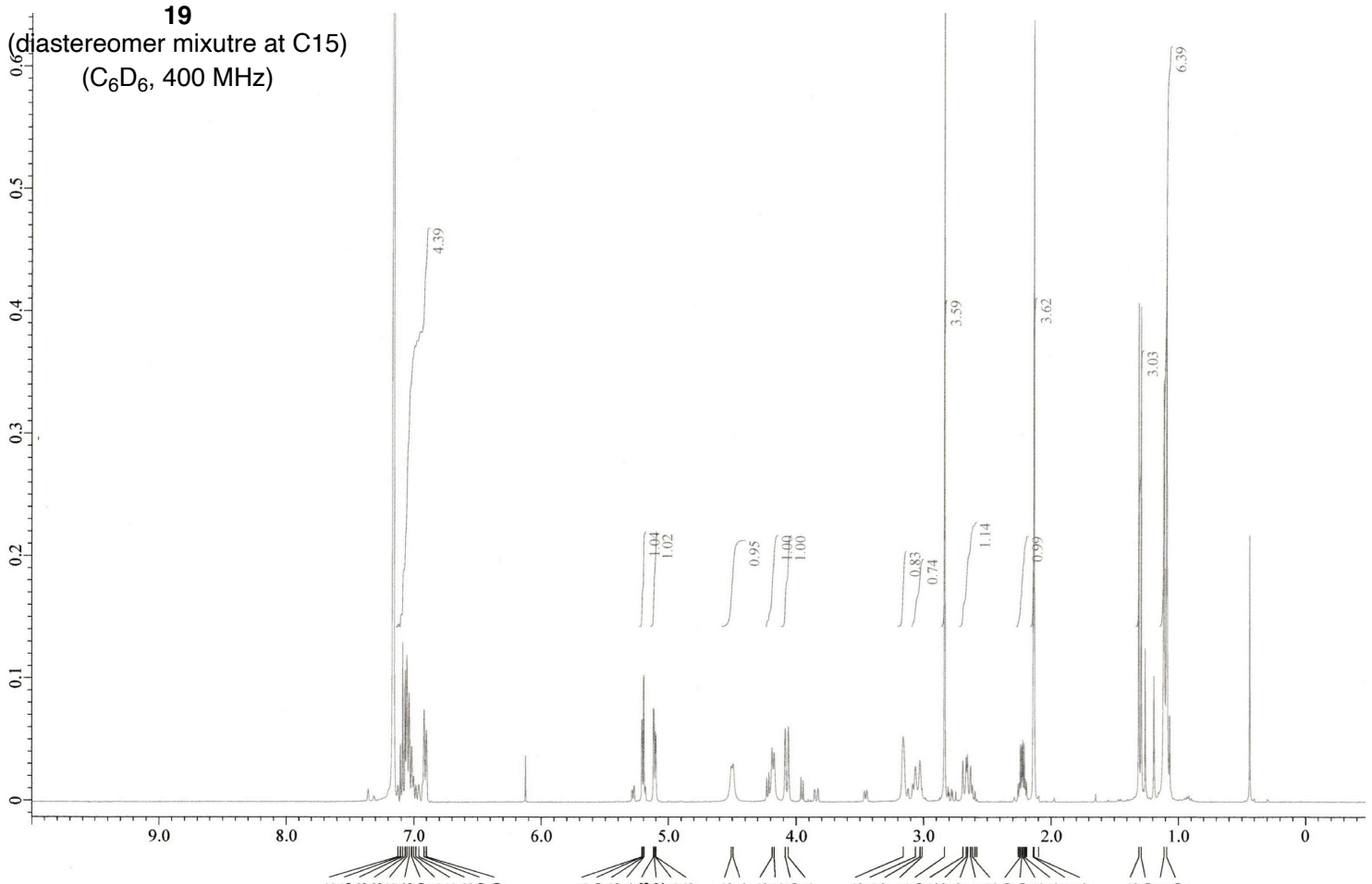

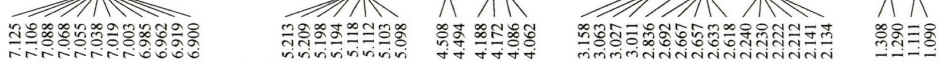

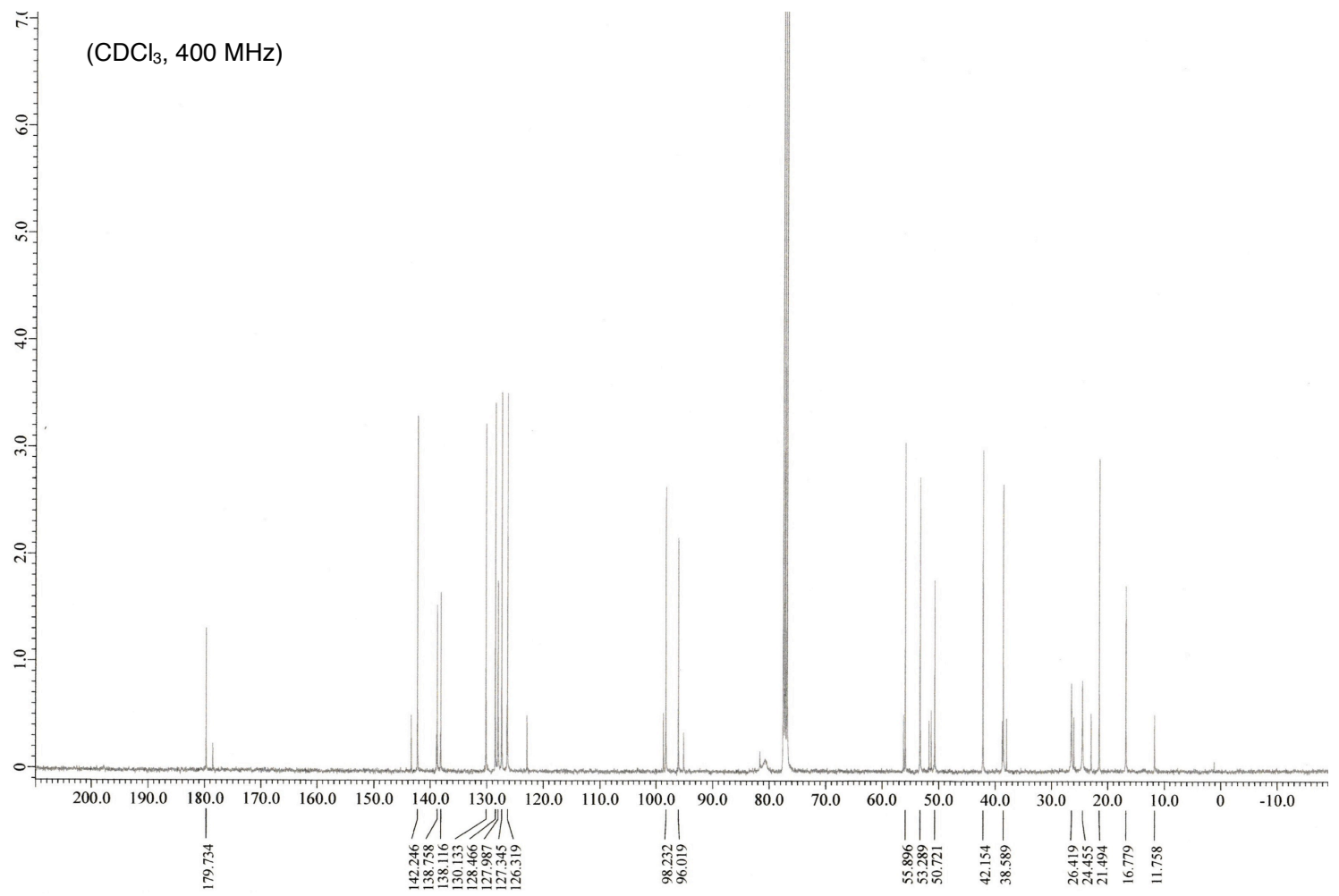



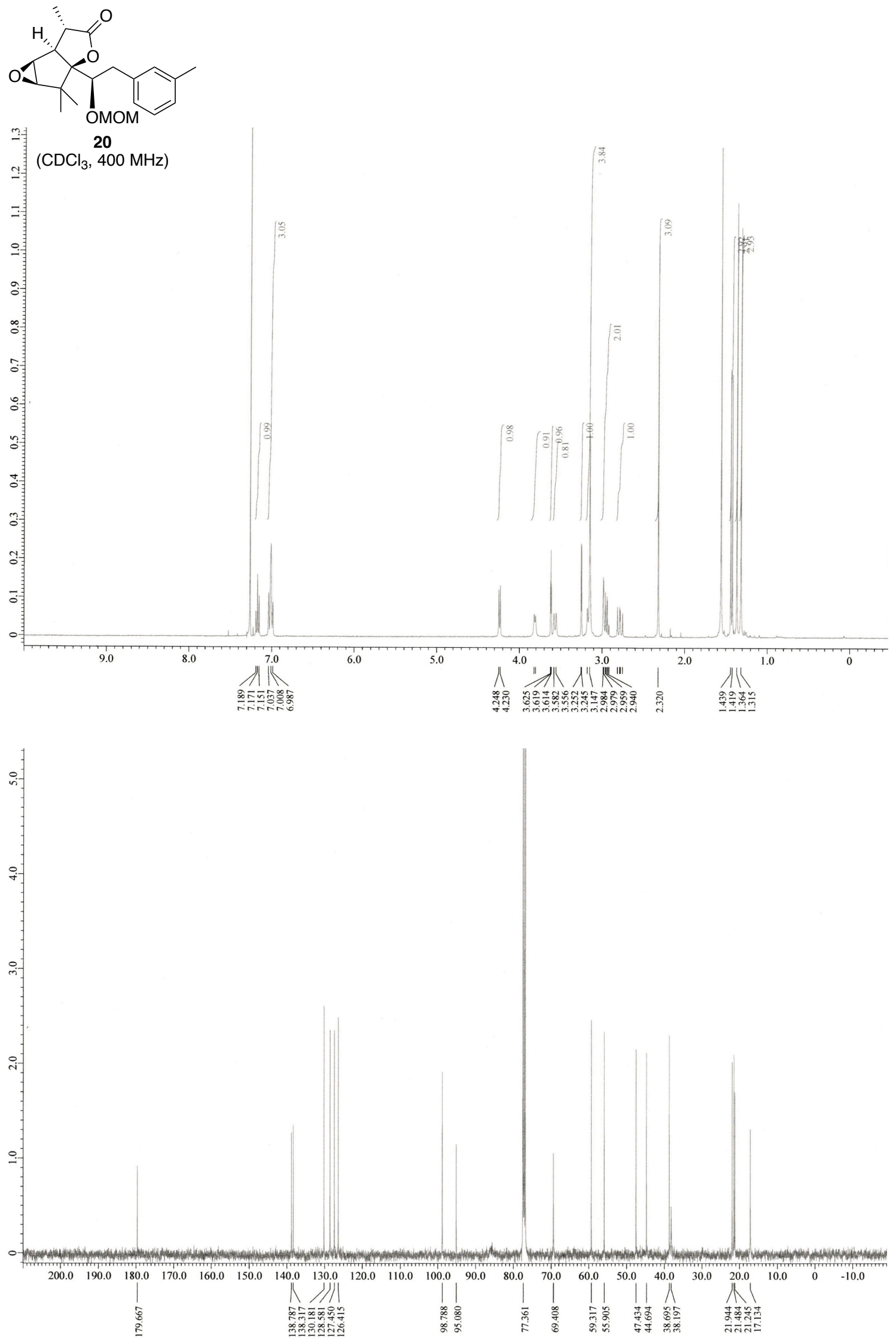

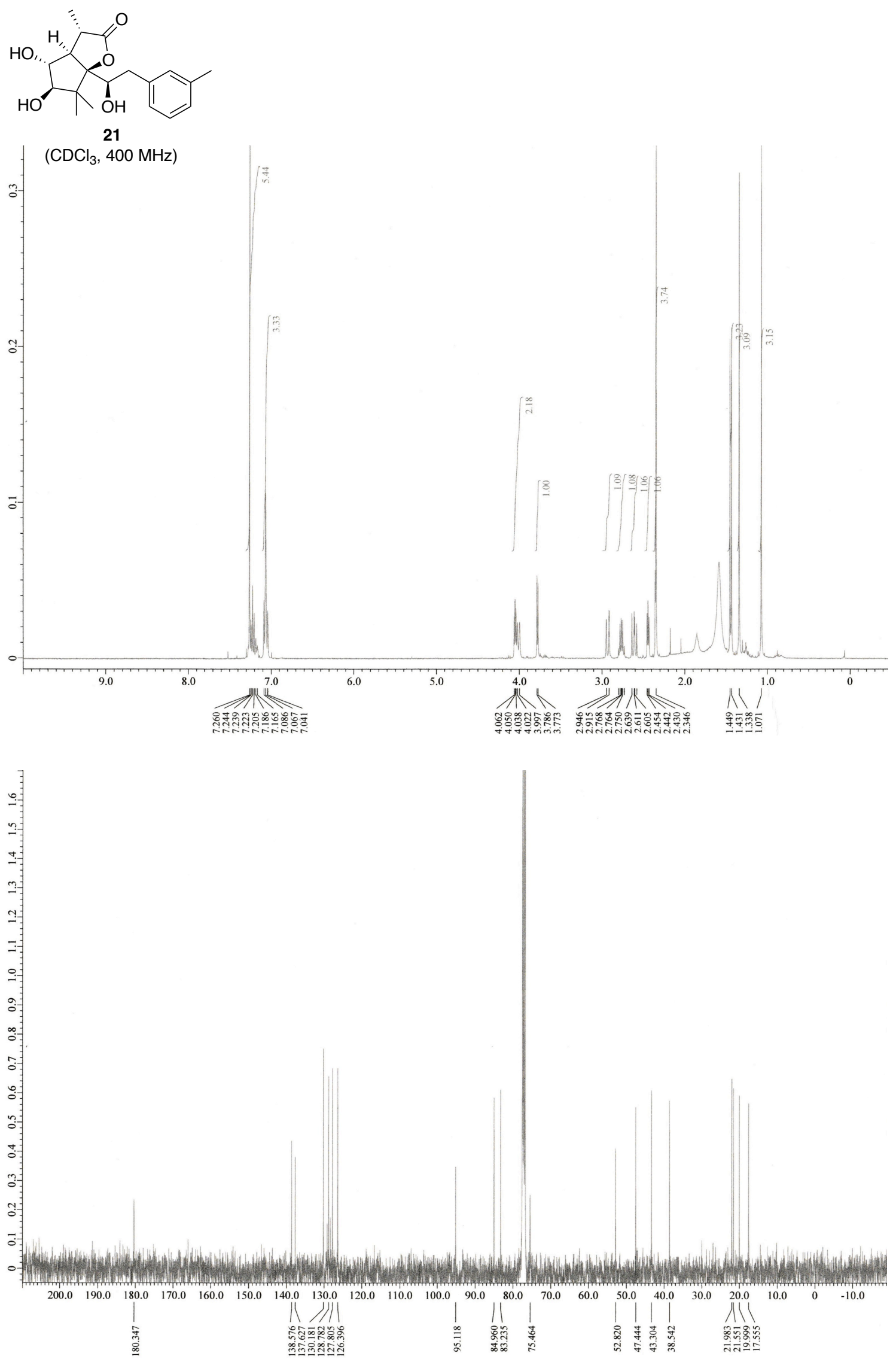

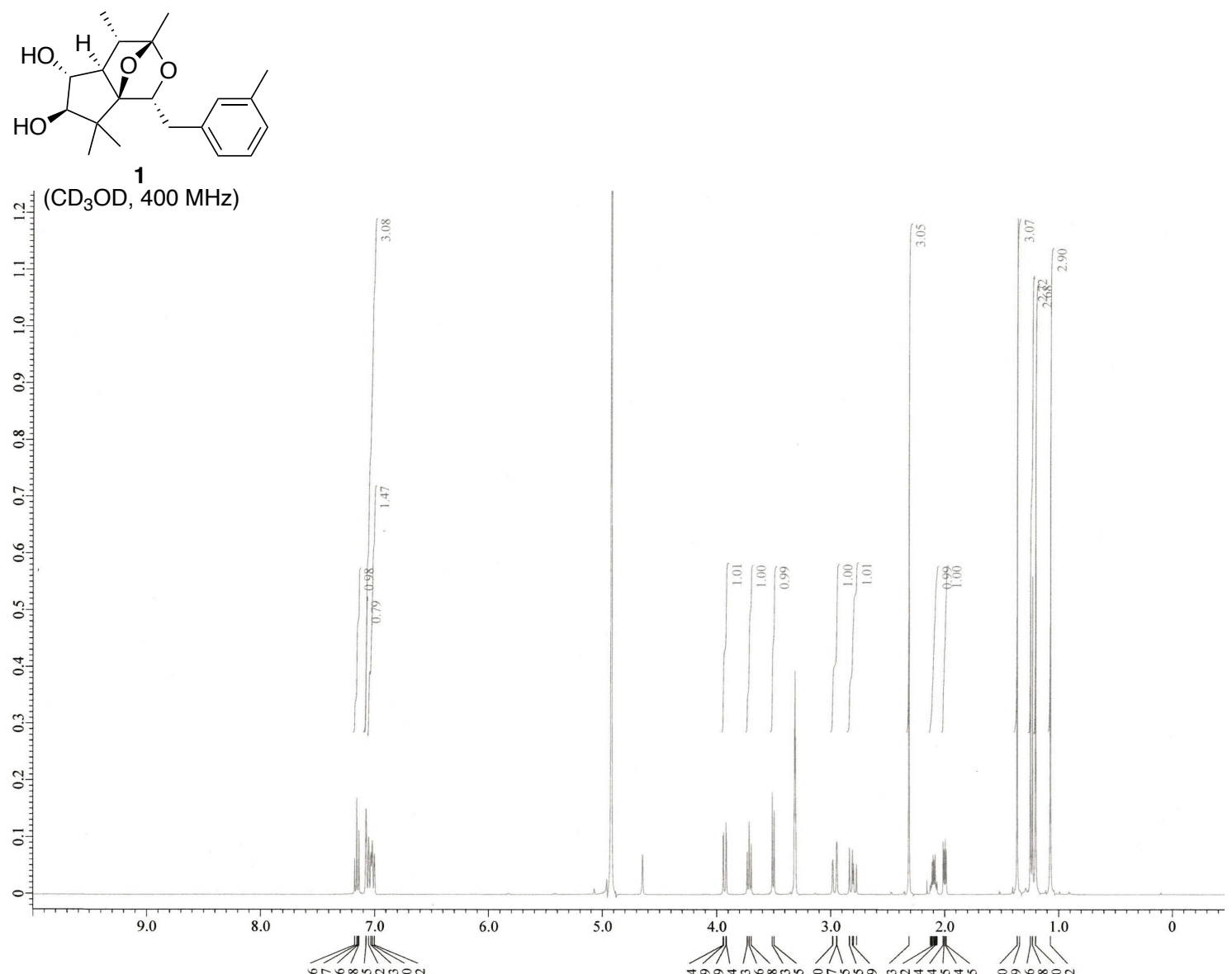

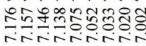

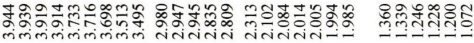

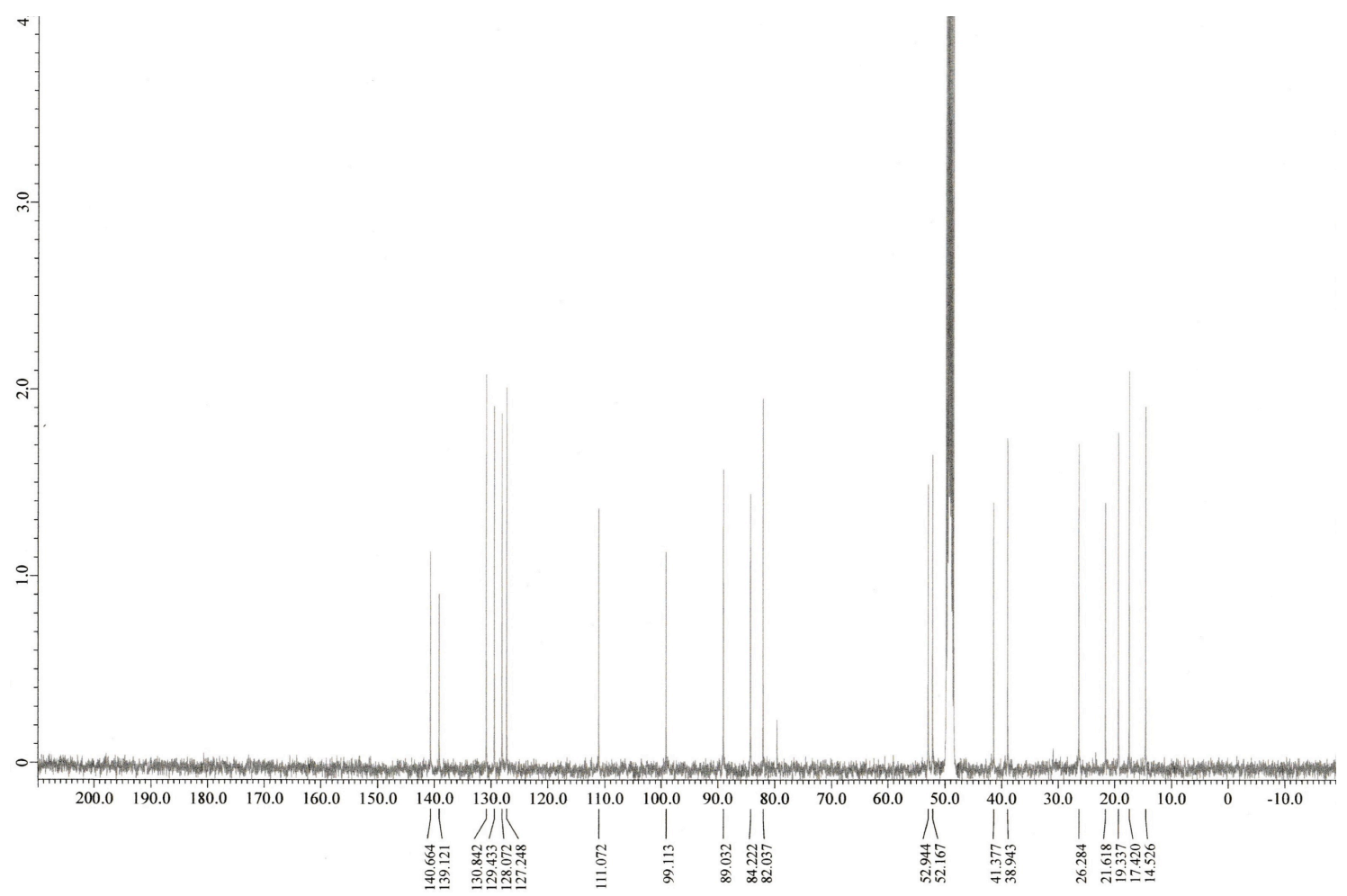


X-ray single-crystal diffraction data (relative configuration of racemic 15) : CCDC 2019833

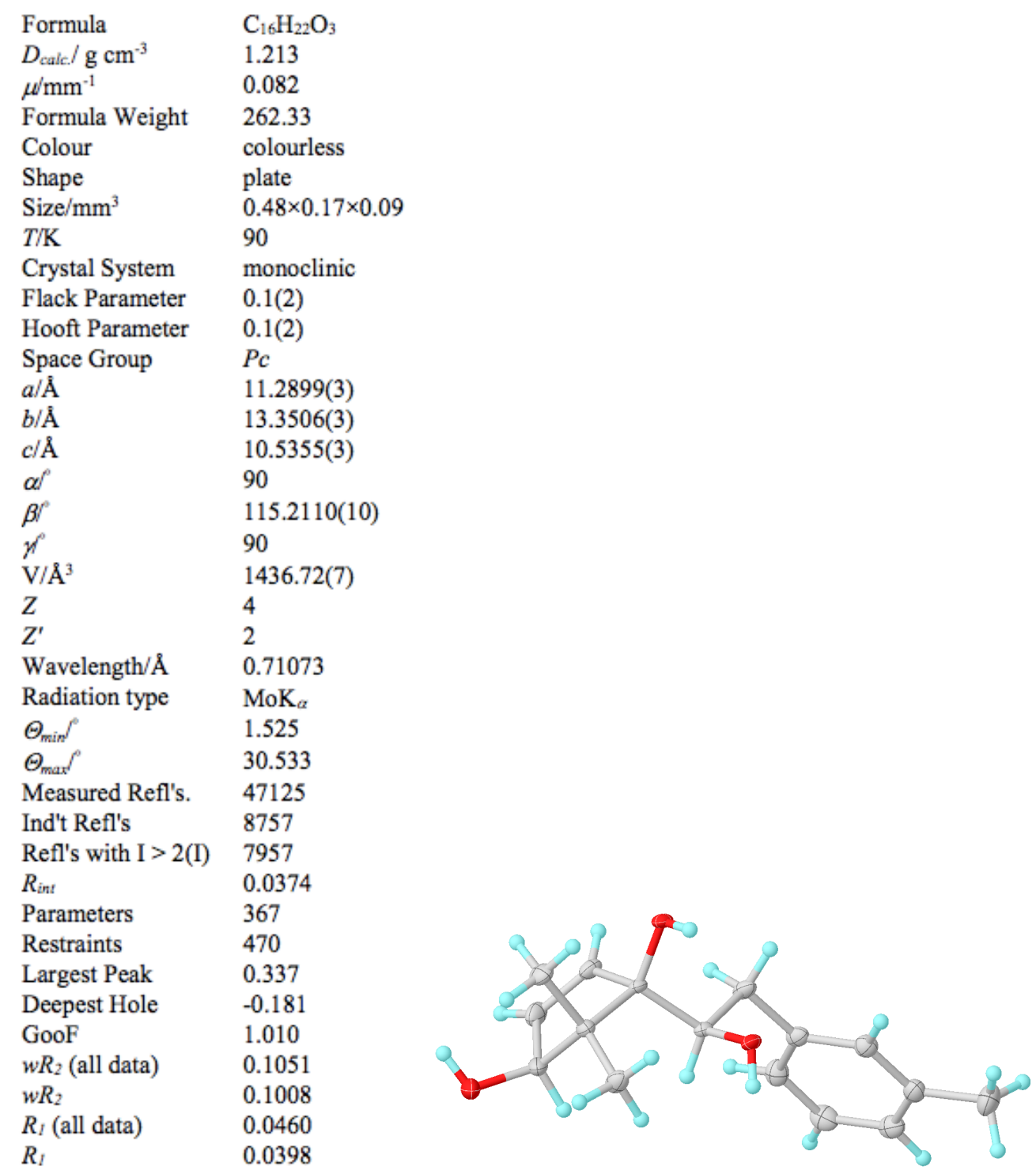


X-ray single-crystal diffraction data (relative configuration of racemic 20) : CCDC 2019835

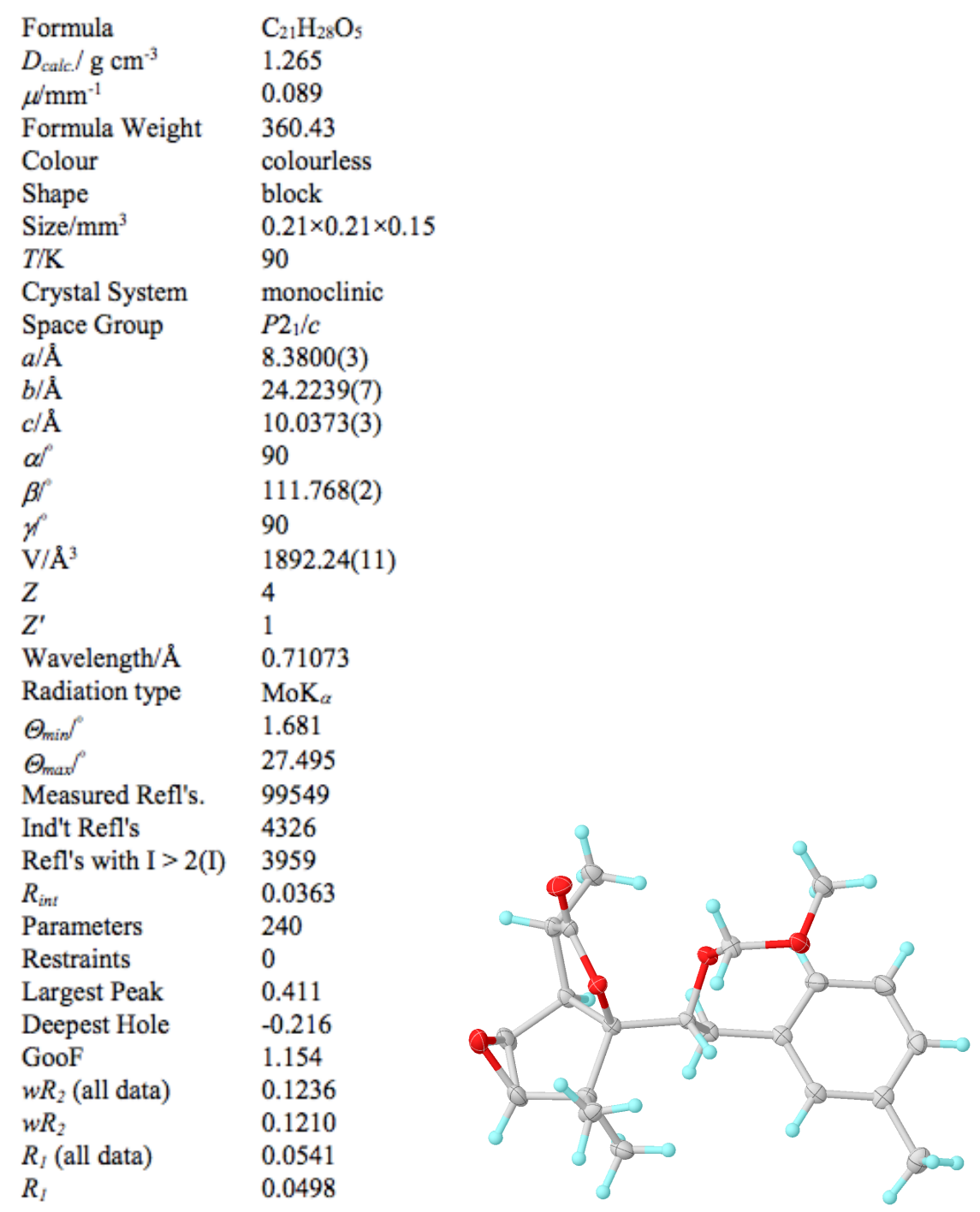


comparison of ${ }^{1} \mathrm{H}$ and ${ }^{13} \mathrm{C}$ NMR data for natural mollebenzylanol $\mathrm{A}$ and synthetic mollebenzylanol $\mathrm{A}$ in $\mathrm{CD}_{3} \mathrm{OD}$

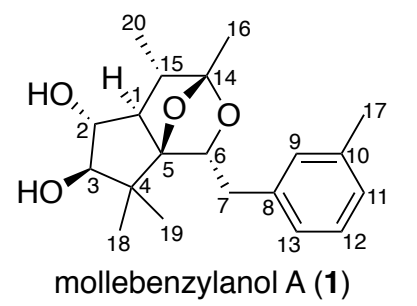

\begin{tabular}{|c|c|c|c|c|}
\hline & \multicolumn{2}{|c|}{ proton $(J$ in $\mathrm{Hz})$} & \multicolumn{2}{|c|}{ carbon } \\
\hline No. & synthetic & natural & synthetic & natural \\
\hline 1 & $2.00, \mathrm{dd}(7.8,3.7)$ & $2.00, \mathrm{dd}(7.5,3.6)$ & 52.9 & 53.0 \\
\hline 2 & $3.72, \mathrm{~m}$ & $3.72, \mathrm{dd}(7.5,6.6)$ & 84.2 & 84.3 \\
\hline 3 & $3.50, \mathrm{~d}(6.9)$ & $3.50, \mathrm{~d}(6.6)$ & 89.0 & 89.1 \\
\hline 4 & & & 41.4 & 41.4 \\
\hline 5 & & & 99.1 & 99.2 \\
\hline 6 & $3.93, \mathrm{dd}(10.1,1.8)$ & $3.93, \mathrm{dd}(10.1,1.7)$ & 82.0 & 82.0 \\
\hline $7 \mathrm{a}$ & $2.96, \mathrm{dd}(14.2,0.9)$ & $2.96, \mathrm{dd}(14.2,1.7)$ & 38.9 & 39.0 \\
\hline $7 b$ & $2.82, \mathrm{dd}(14.2,10.5)$ & $2.82, \mathrm{dd}(14.2,10.1)$ & & \\
\hline 8 & & & 140.7 & 140.7 \\
\hline 9 & 7.08, br s & 7.07 , br s & 130.8 & 130.9 \\
\hline 10 & & & 139.1 & 139.1 \\
\hline 11 & 7.01, br d (7.3) & 7.01, br d (7.6) & 128.1 & 128.1 \\
\hline 12 & $7.15, \mathrm{~m}$ & $7.15, \mathrm{t}(7.6)$ & 129.4 & 129.4 \\
\hline 13 & 7.04, br d, (7.6) & 7.04, br d, (7.6) & 127.2 & 127.3 \\
\hline 14 & & & 111.1 & 111.1 \\
\hline 15 & $2.10, \mathrm{qd}(7.0,3.7)$ & $2.10, \mathrm{qd}(7.0,3.6)$ & 52.2 & 52.2 \\
\hline 16 & $1.36, \mathrm{~s}$ & $1.36, \mathrm{~s}$ & 17.4 & 17.4 \\
\hline 17 & $2.31, \mathrm{~s}$ & $2.31, \mathrm{~s}$ & 21.6 & 21.6 \\
\hline 18 & $1.07, \mathrm{~s}$ & $1.07, \mathrm{~s}$ & 19.3 & 19.3 \\
\hline 19 & $1.20, \mathrm{~s}$ & $1.20, \mathrm{~s}$ & 26.3 & 26.3 \\
\hline 20 & $1.24, \mathrm{~d}(7.3)$ & $1.24, \mathrm{~d}(7.0)$ & 14.5 & 14.5 \\
\hline
\end{tabular}

